\title{
Couplings to Classical and Non-Classical Squeezed White Noise as Stationary Markov Processes
}

\author{
By \\ Jürgen Hellmich, Reinhard Honegger,* Claus Köstler, ${ }^{*}$ \\ Burkhard KÜMMERER*** and Alfred RIECKERS**
}

\begin{abstract}
The squeezed white noise states of the quantum optics literature are identified with specific quasifree states on the bosonic $\mathrm{C}^{*}$-Weyl (resp. CCR) algebra, which are not gauge invariant. Their properties are discussed, and concrete realizations of their GNS-representations are given. The squeezed white noise states are obtained from the chaotic temperature (or white noise states) by the application of squeezing Bogoliubov transformations, which may lead to a noise reduction in selected modes. A squeezing strength is determined, below which the squeezed white noise states remain classical and above which they are rendered non-classical. We further couple additional systems to the squeezed white noise, the quantum character of which are leading to a large class of stationary quantum Markov processes with related quantum stochastic calculus. Their interactions are described in terms of unitary cocycles, which arise from adapted additive cocycles by means of solutions of stochastic differential equations resp. by stochastic Itô integrals. The restriction of the Markovian dynamics to the sub-system leads to a quantum semi-group, for which the Lindblad generator is of detailed balance type. Finally the stochastic Itô table for squeezed white noise is derived. From the latter non-classicality of the squeezed white noise process can now be checked by inspection.
\end{abstract}

\section{$\S 1 . \quad$ Introduction}

Squeezed states constitute nowadays the main class of experimentally

\footnotetext{
Communicated by Y. Takahashi. Received June 15, 2000.

2000 Mathematics Subject Classification(s): 81S25, 60H40, 81V10, 81V80.

*Mathematisches Institut, Universität Tübingen, D-72076 Tübingen, Germany.

** Institut für Theoretische Physik, Universität Tübingen, D-72076 Tübingen, Germany.

*** Mathematisches Institut A, Universität Stuttgart, D-70569 Stuttgart, Germany.
} 
preparable non-classical states of the photon field, exhibiting interesting quantum correlation and fluctutation effects [55], [56], [57], [58], [59], [54], [40], [29], [41], [14]. Recently we have investigated under which squeezing Bogoliubov transformations some frequently used optical states (namely, the quasifree, the classical, and the coherent states) acquire a non-classical generating function [26]. In [27] the classicality, resp. non-classicality of photon states has been determined by comparing the field fluctuations before and after a squeezing Bogoliubov transformation. These investigations (cf., also [25], [28] and references therein) have been performed in terms of algebraic quantum field theory, based on the smeared Weyl elements which generate the $\mathrm{C}^{*}$-algebraic Weyl algebra. For certain physical applications also non-Fock representations of the Weyl algebra come into play. Especially the collective and coherence properties lead to nontrivial dependences of the field resp. creation operators on the representation space. The test functions of the smeared creation operators are intimately connected with the photon wave funtions and usually depend on both the space and the time coordinates. In any case, the choice of test function space determines the abstract Weyl algebra — but not its representation - uniquely.

In the quantum optics literature [17], [10], squeezing of light is often considered from a stochastic point of view. There, the squeezed white noise states are postulated by means of their correlation functionals. A formal Itô table makes it possible to obtain the Langevin and Master equations.

In the $80 \mathrm{~s}$, theories for a mathematically rigorous formulation of quantum (i.e., non-commutative) stochastic differential equations have been developed, e.g. [11], [31], [30]. Instead of the classical Brownian motion as integrand there have been taken the annihilation and creation operators on the symmetric (resp. antisymmetric) Fock space, which refer to the algebra of the canonical commutation (resp. anticommutation) relations. These Fock space operators are in fact generalized quantum stochastic processes, which have to be smeared with smooth test functions over the time axis. The connection with the corresponding operators of quantum field theory may be established by using the spectral theorem for the one-photon Hamiltonian and restricting the one-photon wave functions to a fixed direction of polarization. Keeping this in mind we may use the results of the cited quantum field theoretic squeezing theory for the present quantum stochastic considerations.

A treatment of non-commutative Markov processes, which is more general than the Fock space formalism, has been elaborated in [34], [35], [36], where the stochastic environment of a quantum system is identified with a so-called 
generalized white noise. Especially, the well-known non-squeezed white noise states are examples for the generalized white noise from [34], [35], [36]. In [30] a mathematically rigorous stochastic integration has been derived for nonsqueezed white noise states (chaotic temperature state) within the scheme of a Hudson-Parthasarathy stochastic integration.

The present work is devoted to a mathematically rigorous investigation of the squeezed white noise states and the development of an associated quantum stochastic calculus, which is sufficient for the construction of quantum Markov processes. The paper is separated into two parts. In the first part (Section 2) the squeezed white noise states are investigated, whereas in the second part (Section 3) the non-commutative stochastic calculus from our recent work [19] is applied to the coupling of squeezed white noise to a further system $\mathcal{A}_{0}$, leading to the tensor product algebra $\mathcal{A}_{0} \otimes \mathcal{C}$ of the total interacting system, where $\mathcal{C}$ is the weak closure of the GNS-represented Weyl algebra.

In Subsection 2.1 the squeezed white noise states from [17], [10] are interpreted as specific quasifree states in the operator algebraic setup concerning the bosonic $\mathrm{C}^{*}$-Weyl (resp. CCR) algebra $\mathcal{W}\left(L^{2}(\mathbb{R})\right.$ ) over $L^{2}(\mathbb{R})$. In Subsection 2.2 we verify with the help of [25], [28] that the squeezed white noise states are obtained from the chaotic temperature states by squeezing Bogoliubov transformations on $\mathcal{W}\left(L^{2}(\mathbb{R})\right)$. With the aid of these Bogoliubov transformations several properties of the squeezed white noise states are deduced from those of the chaotic temperature states (Subsection 2.3). The squeezing procedures of the white noise states also ensure a specific noise reduction in certain photon field modes.

Then in Subsection 2.4 the classicality resp. non-classicality of the squeezed white noise states is investigated. Using results from [26], [27], a squeezing strength is determined above which all squeezed white noise states are rendered non-classical and below which they remain classical. Classicality resp. nonclassicality of the squeezed white noise is also expressed by a comparision of its fluctuations with those of the vacuum. Finally in Subsection 2.5 we deduce a concrete realization of the GNS-representations of squeezed white noise in terms of the "Araki-Woods" construction, that is in terms of a tensor product of two Fock spaces.

In Subsection 3.1 the squeezed white noise states are shown to be further examples of the above mentioned generalized white noise from [35]. In Subsections 3.2 and 3.3 those couplings to the mentioned additional system $\mathcal{A}_{0}$ are completely specified, which lead to stationary quantum Markov processes. The necessary quantum stochastic calculus is developed in our recent work [19] with 
the help of Hilbert module techniques. The unitary cocycle necessary for the coupling to white noise, which determines the Markov process, arises uniquely from an adapted additive cocycle - quantum Brownian motion - as increment process for the stochastic Itô integral (solution of a stochastic differential equation), where the latter has to obey a condition for its mutual quadratic variation, and conversely. Observe that nowadays Hilbert modules [38] play an increasingly important role in the context of quantum stochastic processes, cf., e.g., [2], [3], [50], [18].

Subsection 3.4 is devoted to Markovian couplings, which arise from canonical adapted additive cocycles of the type

$$
\beta_{t}=M^{*} \otimes a(t)-M \otimes a^{*}(t)+K \otimes \mathbb{1} t, \quad t \geq 0,
$$

(cf. Equation (3.15)), which includes the well-known annihilation and creation expressions $a(t)$ resp. $a^{*}(t)$ for the squeezed white noise ( $t$ time parameter) from the quantum optics literature [17], [10] (cf. also [31]), and where the operators $K$ and $M$ act on the subsystem $\mathcal{A}_{0}$. The reduction of the so obtained stationary quantum Markov processes to the coupled subsystem $\mathcal{A}_{0}$ leads to an irreversible stationary dynamical system, the Lindblad generator of which is proven to be of detailed balance type.

In Subsection 3.5 a derivation of the Itô table for the squeezed white noise is carried through. The coefficients of the Itô table form a certain $3 \times 3$-matrix, in which only the first $2 \times 2$-sub-matrix may have non-trivial entries, which are expressed by two variable complex numbers. Our investigation shows, that the table describes a well defined squeezed white noise process on the Weyl algebra, if and only if the $2 \times 2$-matrix has negative determinant. It shows also that this process is non-classical, if and only if the absolute values of the non-diagonal elements are smaller or equal to those of the diagonal elements. Also in this respect it supplements the existing literature. (For a treatment of the Itô table in the thermo field language cf., e.g., [7], [48].)

Finally we give a formal derivation of the singular interaction operator of the total system, which corresponds to the interacting unitary cocycle arising from Equation (1.1), and which is in accordance with [8], [9].

\section{§2. Squeezed White Noise}

\section{§2.1. Squeezed white noise states}

In the quantum optical literature [17] the states $\omega_{n, c}$ of the squeezed white noise are given by their expectation values of the creation and annihilation 
operators, $a_{n, c}^{*}(f)$ resp. $a_{n, c}(f)$ for testfunctions $f$ from the complex Hilbert space $L^{2}(\mathbb{R})$ of square integrable complex-valued functions. In the smeared field formalism with the usual scalar product $\langle f \mid g\rangle=\int_{\mathbb{R}} \overline{f(t)} g(t) \mathrm{d} t$ and the natural complex conjugation $J f=\bar{f}$ on $L^{2}(\mathbb{R})$ these expectations are postulated for $n \geq 0$ and $c \in \mathbb{C}$ to be

$$
\begin{aligned}
\left\langle\omega_{n, c} ; a_{n, c}(f)\right\rangle & =\left\langle\omega_{n, c} ; a_{n, c}^{*}(f)\right\rangle=0, \\
\left\langle\omega_{n, c} ; a_{n, c}(f) a_{n, c}^{*}(g)\right\rangle & =(n+1)\langle f \mid g\rangle, \\
\left\langle\omega_{n, c} ; a_{n, c}^{*}(f) a_{n, c}(g)\right\rangle & =n\langle J f \mid J g\rangle=n\langle g \mid f\rangle, \\
\left\langle\omega_{n, c} ; a_{n, c}(f) a_{n, c}(g)\right\rangle & =c\langle f \mid J g\rangle, \\
\left\langle\omega_{n, c} ; a_{n, c}^{*}(f) a_{n, c}^{*}(g)\right\rangle & =\bar{c}\langle J f \mid g\rangle,
\end{aligned}
$$

for arbitrary $f, g \in L^{2}(\mathbb{R})$. The squeezed white noise states are assumed to be quasifree, and thus the expectations of the higher order correlations are uniquely determined by the above ones. Furthermore, in [16], [17] it is shown that the uncertainty relations imply the condition $n(n+1) \geq|c|^{2}$.

The aim of the present subsection is to realize the above postulated squeezed white noise states as (abstract) states on the $\mathrm{C}^{*}$-Weyl algebra $\mathcal{W}\left(L^{2}(\mathbb{R})\right)$ of the exponentiated canonical commutation relations (CCR). The Weyl algebra $\mathcal{W}\left(L^{2}(\mathbb{R})\right)$ over $L^{2}(\mathbb{R})$ is uniquely generated by the unitary Weyl operators $W(f), f \in L^{2}(\mathbb{R})$, satisfying the Weyl relations (for $\hbar=1$, cf. [12, Theorem 5.2.8])

$$
\begin{aligned}
W(f) W(g) & =\exp \left\{-\frac{i}{2} \operatorname{Im}\langle f \mid g\rangle\right\} W(f+g), \\
W(f)^{*} & =W(-f), \quad f, g \in L^{2}(\mathbb{R}) .
\end{aligned}
$$

The state space of $\mathcal{W}\left(L^{2}(\mathbb{R})\right)$ is denoted by $\mathcal{S}$.

The general notion of a quasifree state (a state whose higher-order truncated functionals vanish) was introduced in [47], [49] (cf. also [12, p. $40 \mathrm{f}$ ], [23]). Let us denote by $\mathcal{S}_{\mathrm{qf}}$ the set of all quasifree states on $\mathcal{W}\left(L^{2}(\mathbb{R})\right)$. We do not give the original definition, however we note that the characteristic function for each $\omega \in \mathcal{S}_{\mathrm{qf}}$ is of the form

$$
\langle\omega ; W(f)\rangle=\exp \left\{i \ell(f)-\frac{1}{4} s(f, f)\right\}, \quad \forall f \in L^{2}(\mathbb{R}),
$$

where $\ell: L^{2}(\mathbb{R}) \rightarrow \mathbb{R}$ is a real-linear form and $s: L^{2}(\mathbb{R}) \times L^{2}(\mathbb{R}) \rightarrow \mathbb{R}$ is a positive symmetric real-bilinear form satisfying

$$
|\operatorname{Im}\langle f \mid g\rangle|^{2} \leq s(f, f) s(g, g), \quad \forall f, g \in L^{2}(\mathbb{R}),
$$


[5], [4], [53], [42], [23], [24], [27] (cf. also [6]). Especially, each $\omega \in \mathcal{S}_{\mathrm{qf}}$ is entireanalytic, and thus in its GNS-representation $\left(\Pi_{\omega}, \mathcal{H}_{\omega}, \Omega_{\omega}\right)$ (cf. e.g. [51, Definition I.9.15]) the existence of the selfadjoint field operators $\Phi_{\omega}(f), f \in L^{2}(\mathbb{R})$ with $\Pi_{\omega}(W(t f))=\exp \left\{i t \Phi_{\omega}(f)\right\} \forall t \in \mathbb{R}$, is ensured by Stone's theorem. The annihilation and creation operators, $a_{\omega}(f):=2^{-1 / 2}\left(\Phi_{\omega}(f)+i \Phi_{\omega}(i f)\right)$ resp. $a_{\omega}^{*}(f):=2^{-1 / 2}\left(\Phi_{\omega}(f)-i \Phi_{\omega}(i f)\right)$, associated with $\omega$ fulfill the CCR $\left[a_{\omega}(f), a_{\omega}(g)\right]=\left[a_{\omega}^{*}(f), a_{\omega}^{*}(g)\right]=0$ and $\left[a_{\omega}(f), a_{\omega}^{*}(g)\right]=\langle f \mid g\rangle \mathbb{1}$. Moreover, $f \in L^{2}(\mathbb{R}) \mapsto \Phi_{\omega}(f)$ is real-linear, $f \mapsto a_{\omega}(f)$ is (complex-) antilinear and $f \mapsto a_{\omega}^{*}(f)$ is (complex-) linear. The cyclic vector $\Omega_{\omega}$ is contained in the domain of each polynomial of the field, resp., creation and annihilation operators, and we briefly write $\left\langle\omega ; \Phi_{\omega}\left(f_{1}\right) \cdots \Phi_{\omega}\left(f_{k}\right)\right\rangle$ for the scalar product $\left\langle\Omega_{\omega} \mid \Phi_{\omega}\left(f_{1}\right) \cdots \Phi_{\omega}\left(f_{k}\right) \Omega_{\omega}\right\rangle$ in $\mathcal{H}_{\omega}$ (cf., e.g., [12, Subsection 5.2.3]). The field, creation and annihilation operators in the representation of the squeezed white noise state $\omega_{n, c}$ are simply indexed by $n, c$, writing $\Phi_{n, c}(f), a_{n, c}^{*}(f)$, resp. $a_{n, c}(f)$, for test functions $f \in L^{2}(\mathbb{R})$.

Differentiating $\mathbb{R} \ni t \mapsto\langle\omega ; W(t f)\rangle$ from Equation (2.7) leads to the cummulants $\left\langle\omega ; \Phi_{\omega}(f)^{k}\right\rangle$ for $k \in \mathbb{N}$. Especially, we obtain the fluctuations (variances) of the field operators $\Phi_{\omega}(f), f \in L^{2}(\mathbb{R})$, to be [26], [27]

$$
\operatorname{Var}\left(\omega ; \Phi_{\omega}(f)\right):=\left\langle\omega ; \Phi_{\omega}(f)^{2}\right\rangle-\left\langle\omega ; \Phi_{\omega}(f)\right\rangle^{2}=\frac{1}{2} s(f, f) .
$$

Theorem 2.1. For $n \in \mathbb{R}$ and $c \in \mathbb{C}$ consider the symmetric realbilinear form $s_{n, c}$ on $L^{2}(\mathbb{R})$ given by

$$
s_{n, c}(f, g):=(2 n+1) \operatorname{Re}\langle f \mid g\rangle+2 \operatorname{Re}(c\langle f \mid J g\rangle), \quad \forall f, g \in L^{2}(\mathbb{R}) .
$$

Then there exists a unique $\omega_{n, c} \in \mathcal{S}$ with the characteristic function

$$
\left\langle\omega_{n, c} ; W(f)\right\rangle=\exp \left\{-\frac{1}{4} s_{n, c}(f, f)\right\}, \quad \forall f \in L^{2}(\mathbb{R}),
$$

if and only if $n(n+1) \geq|c|^{2}$ and $n \geq 0$, or equivalently, if and only if $n \geq$ $\sqrt{|c|^{2}+1 / 4}-1 / 2$. The state $\omega_{n, c}$ is the unique quasifree state on $\mathcal{W}\left(L^{2}(\mathbb{R})\right)$ satisfying the expectation relations (2.1) to (2.5) with its associated creation and annihilation operators. The state $\omega_{n, c}$ is a factor state on $\mathcal{W}\left(L^{2}(\mathbb{R})\right)$.

Proof. Immediate consequence of the mentioned results concerning quasifree states and the condition (2.8).

Let us call for $c \neq 0$ the state $\omega_{n, c}$ a squeezed white noise state, and for $c=0, \omega_{n, c=0} \equiv \omega_{n}$ a white noise or chaotic temperature state [17], [30], [31], [13], [39], where the specification chaotic is often dropped but should be kept 
in mind, in order to discriminate them from the temperature states of Planck's radiation law [20], [21]. By

$$
\mathcal{S}_{\mathrm{swn}}:=\left\{\omega_{n, c} \in \mathcal{S}_{\mathrm{qf}} \mid c \in \mathbb{C}, n \geq \sqrt{|c|^{2}+1 / 4}-1 / 2\right\}
$$

we introduce the set of all (squeezed) white noise states, and by

$$
\mathcal{S}_{\text {temp }}:=\left\{\omega_{\kappa} \equiv \omega_{\kappa, 0} \in \mathcal{S}_{\text {swn }} \mid \kappa \geq 0\right\}
$$

the set of all (unsqueezed) white noise or temperature states. Especially for $\kappa=0$ we obtain the Fock vacuum state $\omega_{0} \equiv \omega_{\text {vac }}$.

For calculating the fluctuations one considers the modified field expressions

$$
Q_{\phi}(t)=\Phi_{n, c}\left(\mathrm{e}^{-i \phi} \chi_{[0, t]}\right), \quad P_{\phi}(t)=\Phi_{n, c}\left(i \mathrm{e}^{-i \phi} \chi_{[0, t]}\right)=Q_{\phi-\pi / 2}(t), \quad t \geq 0,
$$

with some phase angle $\phi \in\left[0,2 \pi\left[\right.\right.$ and the characteristic function $\chi_{I}$ of the interval $I$. With (2.9) and (2.10) one immediately obtains

$$
\begin{aligned}
& \operatorname{Var}\left(\omega_{n, c} ; Q_{\phi}(t)\right)=\left(n+\frac{1}{2}+\operatorname{Re}\left(c \mathrm{e}^{2 i \phi}\right)\right) t, \\
& \operatorname{Var}\left(\omega_{n, c} ; P_{\phi}(t)\right)=\left(n+\frac{1}{2}-\operatorname{Re}\left(c \mathrm{e}^{2 i \phi}\right)\right) t .
\end{aligned}
$$

Minimizing (2.12) and maximizing (2.13) with respect to the phase angle $\phi$ gives

$$
\begin{aligned}
& \operatorname{Min} \operatorname{Var}\left(\omega_{n, c} ; t\right):=\min _{\phi \in[0,2 \pi[} \operatorname{Var}\left(\omega_{n, c} ; Q_{\phi}(t)\right)=\left(n+\frac{1}{2}-|c|\right) t \\
& \operatorname{Max} \operatorname{Var}\left(\omega_{n, c} ; t\right):=\max _{\phi \in[0,2 \pi[} \operatorname{Var}\left(\omega_{n, c} ; P_{\phi}(t)\right)=\left(n+\frac{1}{2}+|c|\right) t
\end{aligned}
$$

\section{§2.2. Squeezing of the white noise or temperature states}

For each symplectic transformation $T$ on $L^{2}(\mathbb{R})$ (that is, $T: L^{2}(\mathbb{R}) \rightarrow$ $L^{2}(\mathbb{R})$ is bijective, real-linear and satisfies the symplectic condition $\operatorname{Im}\langle T f \mid T g\rangle$ $=\operatorname{Im}\langle f \mid g\rangle \forall f, g \in L^{2}(\mathbb{R})$; thus $T^{-1}$ is symplectic, too) there exists a unique *-automorphism $\alpha_{T}$ on the $\mathrm{C}^{*}$-Weyl algebra $\mathcal{W}\left(L^{2}(\mathbb{R})\right)$ with

$$
\alpha_{T}(W(f))=W(T f), \quad \forall f \in L^{2}(\mathbb{R}),
$$

which is called the Bogoliubov transformation associated with $T$ in the Heisenberg picture. The dual mapping $\nu_{T}:=\alpha_{T}^{*}$ is an affine bijection on the state space $\mathcal{S}$ of $\mathcal{W}\left(L^{2}(\mathbb{R})\right)$,

$$
\left\langle\nu_{T}(\omega) ; A\right\rangle=\left\langle\omega ; \alpha_{T}(A)\right\rangle, \quad \forall \omega \in \mathcal{S}, \quad \forall A \in \mathcal{W}\left(L^{2}(\mathbb{R})\right),
$$


i.e., $\nu_{T}$ denotes the Bogoliubov transformation in the Schrödinger picture. It holds $\left(\nu_{T}\right)^{-1}=\nu_{T^{-1}}$, and $\nu_{T}\left(\mathcal{S}_{\mathrm{qf}}\right)=\mathcal{S}_{\mathrm{qf}}[26]$.

Specific Bogoliubov transformations are the so-called gauge transformations of first kind, i.e., $T=z \mathbb{1}$ with $z \in \mathcal{T}:=\{z \in \mathbb{C}|| z \mid=1\}$. We write $\nu_{z}$ for the associated gauge symmetry transformations on $\mathcal{S}$ in the Schrödinger picture. A state $\omega \in \mathcal{S}$ is called gauge invariant, if $\nu_{z}(\omega)=\omega$ for all $z \in \mathcal{T}$.

Due to [54], [55], [56], [25], [28], [26], and [27], quantum optical squeezing processes are described in terms of specific Bogoliubov transformations. Here we consider the symplectic transformations

$$
T_{s, \theta}:=\cosh (s) \mathbb{1}+\exp \{i \theta\} \sinh (s) J
$$

depending on the parameters $\theta \in[0,2 \pi[$ and $s \geq 0$ [25]. From [25] it also follows that

$$
\left(T_{s, \theta}\right)^{-1}=\cosh (s) \mathbb{1}-\exp \{i \theta\} \sinh (s) J=T_{s, \pi+\theta} .
$$

Let us denote the associated Bogoliubov transformation (squeezing operation) by $\nu_{s, \theta}$ in the Schrödinger picture and by $\alpha_{s, \theta}$ in the Heisenberg picture.

Proposition 2.2. For every $s \geq 0$ and $\theta \in\left[0,2 \pi\left[\right.\right.$ it holds $\nu_{s, \theta}\left(\mathcal{S}_{\mathrm{swn}}\right)=$ $\mathcal{S}_{\text {swn }}$.

Proof. Since $\left\langle\nu_{s, \theta}(\omega) ; W(f)\right\rangle=\left\langle\omega ; W\left(T_{s, \theta} f\right)\right\rangle \forall f \in L^{2}(\mathbb{R}) \forall \omega \in \mathcal{S}$ it follows from the equations (2.10) and (2.11) that $\nu_{s, \theta}\left(\omega_{n, c}\right) \in \mathcal{S}_{\text {swn }}$, that is $\nu_{s, \theta}\left(\mathcal{S}_{\text {swn }}\right) \subseteq \mathcal{S}_{\text {swn }}$, and from equation $(2.17)$ that $\left(\nu_{s, \theta}\right)^{-1}\left(\mathcal{S}_{\text {swn }}\right) \subseteq \mathcal{S}_{\text {swn }}$.

We want to realize, however, each squeezed white noise state $\omega_{n, c}$ with $c \neq 0$ as a Bogoliubov transformed white noise state $\omega_{\kappa}$ via some squeezing transformation $\nu_{s, \theta}$. Let us recall that the characteristic function of the temperature state $\omega_{\kappa} \in \mathcal{S}_{\text {temp }}, \kappa \geq 0$, is given by

$$
\left\langle\omega_{\kappa} ; W(f)\right\rangle=\exp \left\{-\frac{2 \kappa+1}{4}\|f\|^{2}\right\}, \quad \forall f \in L^{2}(\mathbb{R}) .
$$

Theorem 2.3. Let $n \geq 0$ and $c \in \mathbb{C}$, satisfying $n(n+1) \geq|c|^{2}$, be given with associated (squeezed) white noise state $\omega_{n, c} \in \mathcal{S}_{\mathrm{swn}}$. Then there uniquely exist the parameters $s \geq 0, \theta \in[0,2 \pi$ [, and a unique $\kappa \geq 0$ with associated white noise or temperature state $\omega_{\kappa} \in \mathcal{S}_{\text {temp }}$, such that

$$
\nu_{s, \theta}\left(\omega_{\kappa}\right)=\omega_{n, c}
$$


Moreover, the parameters are uniquely given as follows:

$$
\begin{aligned}
& s=\frac{1}{2} \operatorname{artanh}\left(\frac{2|c|}{2 n+1}\right), \\
& \theta=\arg (c), \\
& \kappa=\sqrt{(n+1 / 2)^{2}-|c|^{2}}-\frac{1}{2},
\end{aligned}
$$

especially we have $\kappa=0$, if and only if $n(n+1)=|c|^{2}$. Consequently, this result implies that $\mathcal{S}_{\mathrm{swn}}=\left\{\nu_{s, \theta}\left(\omega_{\kappa}\right) \mid s, \kappa \geq 0, \theta \in\left[0,2 \pi[\}=\left\{\nu_{s, \theta}\left(\mathcal{S}_{\text {temp }}\right) \mid s \geq 0\right.\right.\right.$, $\theta \in[0,2 \pi[\}$.

Conversely, let the parameters $s \geq 0, \theta \in[0,2 \pi[$ and the white noise or temperature state $\omega_{\kappa} \in \mathcal{S}_{\mathrm{temp}}, \kappa \geq 0$, be given. Then $\nu_{s, \theta}\left(\omega_{\kappa}\right)=\omega_{n, c}$ with

$$
n=(\kappa+1 / 2) \cosh (2 s)-1 / 2, \quad c=\exp \{i \theta\}(\kappa+1 / 2) \sinh (2 s) .
$$

Proof. Using (2.16) gives

$$
(2 \kappa+1)\left\|T_{s, \theta} f\right\|^{2}=(2 \kappa+1) \cosh (2 s)+\operatorname{Re}(\sinh (2 s)(2 \kappa+1) \exp \{i \theta\}\langle f \mid J f\rangle) .
$$

Comparing with (2.10) yields $2 n+1=(2 \kappa+1) \cosh (2 s)$ and $2 c=\exp \{i \theta\}(2 \kappa+$ $1) \sinh (2 s)$, that is (2.23). The last equation gives $(2.21)$ and $2|c|=(2 \kappa+$ $1) \sinh (2 s)$. Thus dividing the relations implies (2.20). Squaring gives

$$
8|c|^{2}=(2 \kappa+1)^{2}(\cosh (4 s)-1), \quad 8(n+1 / 2)^{2}=(2 \kappa+1)^{2}(\cosh (4 s)+1),
$$

which by insertion yields $(n+1 / 2)^{2}=|c|^{2}+(\kappa+1 / 2)^{2}$, that is (2.22). Addition and subtraction gives

(2.24) $2 n+1+2|c|=(2 \kappa+1) \exp \{2 s\}, 2 n+1-2|c|=(2 \kappa+1) \exp \{-2 s\}$.

The above Theorem ensures the notion of a properly squeezed white noise state $\omega_{n, c}$, whenever we have $c \neq 0$. Especially we have $c=0$, if and only if $s=0$. This implies $\nu_{s, \theta}$ resp. $T_{s, \theta}$ to be a proper squeezing transformation, if and only if $s \neq 0$.

In quantum optics the notion of squeezing is given in terms of diminished field fluctuations for the transformed states, compared with those of the original ones.

Proposition 2.4. Let all be as in Theorem 2.3, especially $\nu_{s, \theta}\left(\omega_{\kappa}\right)=$ $\omega_{n, c}$. Then for every $t>0$ it follows for the field fuctuations that

$$
\begin{aligned}
\operatorname{Min} \operatorname{Var}\left(\omega_{\kappa} ; t\right) & =\operatorname{Max} \operatorname{Var}\left(\omega_{\kappa} ; t\right)=t\left(\kappa+\frac{1}{2}\right), \\
\operatorname{Min} \operatorname{Var}\left(\omega_{n, c} ; t\right) & =t\left(n+\frac{1}{2}-|c|\right)=t\left(\kappa+\frac{1}{2}\right) \exp \{-2 s\}, \\
\operatorname{Max} \operatorname{Var}\left(\omega_{n, c} ; t\right) & =t\left(n+\frac{1}{2}+|c|\right)=t\left(\kappa+\frac{1}{2}\right) \exp \{2 s\} .
\end{aligned}
$$


Consequently, if the squeezing transformation $\nu_{s, \theta}$ is proper (i.e., if $s>0$ or equivalently $c \neq 0)$, then we have that the MinVar- resp. MaxVar-field fluctuations of the squeezed state $\omega_{n, c}=\nu_{s, \theta}\left(\omega_{\kappa}\right)$ are properly diminished resp. enlarged compared with those of the unsqueezed state $\omega_{\kappa}$,

$$
\operatorname{Min} \operatorname{Var}\left(\omega_{n, c} ; t\right)<\operatorname{Min} \operatorname{Var}\left(\omega_{\kappa} ; t\right)=\operatorname{Max} \operatorname{Var}\left(\omega_{\kappa} ; t\right)<\operatorname{Max} \operatorname{Var}\left(\omega_{n, c} ; t\right), \quad t>0
$$

Proof. Equation (2.22) yields

$$
t^{-1} \operatorname{Min} \operatorname{Var}\left(\omega_{\kappa} ; t\right)=t^{-1} \operatorname{Max} \operatorname{Var}\left(\omega_{\kappa} ; t\right)=\kappa+\frac{1}{2}=\sqrt{\left(n+\frac{1}{2}-|c|\right)\left(n+\frac{1}{2}+|c|\right)},
$$

which together with (2.14), (2.15) and (2.24) gives the result.

Let us fix a temperature state $\omega_{\kappa} \in \mathcal{S}_{\text {temp }}$ with some $\kappa \geq 0$. We transform the unsqueezed state $\omega_{\kappa}$ by the Bogoliubov transformations $\nu_{s, \theta}$ with varying parameters $s \geq 0$ and $\theta \in[0,2 \pi[$. By the above Proposition we have the following connection for the field fluctuations before and after the transformation,

$$
\begin{aligned}
& \operatorname{Min} \operatorname{Var}\left(\nu_{s, \theta}\left(\omega_{\kappa}\right) ; t\right)=\operatorname{Min} \operatorname{Var}\left(\omega_{\kappa} ; t\right) \exp \{-2 s\}, \\
& \operatorname{Max} \operatorname{Var}\left(\nu_{s, \theta}\left(\omega_{\kappa}\right) ; t\right)=\operatorname{Max} \operatorname{Var}\left(\omega_{\kappa} ; t\right) \exp \{2 s\} .
\end{aligned}
$$

Hence the MinVar- resp. MaxVar-expressions depend exponentially on the squeezing parameter $s \geq 0$, whereas the phase angle $\theta$ does not show up in these formulas.

\section{§2.3. Geometric characterization}

Here we denote a (squeezed) white noise state $\omega_{n, c}$ faithful, if its normal extension is a faithful state (with unit support) on the GNS von Neumann algebra $\Pi_{n, c}\left(\mathcal{W}\left(L^{2}(\mathbb{R})\right)\right)^{\prime \prime}$, where $\left(\Pi_{n, c}, \mathcal{H}_{n, c}, \Omega_{n, c}\right)$ means the GNS representation of the state $\omega_{n, c} \in \mathcal{S}_{\text {swn }}$.

We now derive some properties and a geometric characterization of the (squeezed) white noise states. The unit ball in $\mathbb{R}^{3}$ is denoted by $B_{1}:=\{x=$ $\left.\left(x_{1}, x_{2}, x_{3}\right) \in \mathbb{R}^{3}|| x \mid \leq 1\right\}$, and the unit sphere is given by $S_{1}:=\left\{x \in B_{1} \mid\right.$ $|x|=1\}$. Furthermore, let us define the half ball $\tilde{B}_{1}:=\left\{x \in B_{1} \mid x_{2}>0\right\}$, the half sphere $\tilde{S}_{1}:=\tilde{B}_{1} \bigcap S_{1}$, and the interior $\tilde{B}_{1}^{o}:=\tilde{B}_{1} \backslash S_{1}$ of the half ball $\tilde{B}_{1}$. Finally, the intersection of an arbitrary subset $\Lambda \subseteq \mathbb{R}^{3}$ with the $x_{2}$-axis denoted by $\ell_{2}$ - is written $\Lambda \cap \ell_{2}$.

Theorem 2.5. Because of the conditions $n(n+1) \geq|c|^{2}$ and $n \geq 0$, the mapping $\omega_{n, c} \mapsto(1 /(n+(1 / 2)))(b,(1 / 2), a)$, where $a:=\operatorname{Re}(c)$ and $b:=\operatorname{Im}(c)$, 
constitutes a one-to-one correspondence between the set $\mathcal{S}_{\mathrm{swn}}$ and the half ball $\tilde{B}_{1}$. It follows that:

(a) The vacuum state $\omega_{0} \equiv \omega_{\text {vac }}$ is represented by the point $(0,1,0) \in$ $\tilde{S}_{1} \cap \ell_{2}$.

(b) $\omega_{n, c} \in \mathcal{S}_{\mathrm{swn}}$ is gauge invariant, if and only if $c=0$. That is, the temperature states $\mathcal{S}_{\text {temp }}$ are the only states in $\mathcal{S}_{\mathrm{swn}}$, which are gauge invariant. We have $\mathcal{S}_{\text {temp }}=\tilde{B}_{1} \cap \ell_{2}$. For different parameters $\kappa \geq 0$ the states $\omega_{\kappa}$ are not quasi-equivalent.

(c) $\tilde{S}_{1}=\left\{\nu_{s, \theta}\left(\omega_{0}\right) \mid s \geq 0, \theta \in\left[0,2 \pi[\}\right.\right.$, and $\tilde{B}_{1}^{o}=\left\{\nu_{s, \theta}\left(\tilde{B}_{1}^{o} \cap \ell_{2}\right) \mid s \geq\right.$ $0, \theta \in[0,2 \pi[\}$.

(d) For $\omega_{n, c} \in \mathcal{S}_{\text {swn }}$ we have the following equivalences:

(i) $n(n+1)=|c|^{2}$;

(ii) $\omega_{n, c}$ corresponds to a point of the half sphere $\tilde{S}_{1}$;

(iii) $\omega_{n, c}$ is a pure state on $\mathcal{W}\left(L^{2}(\mathbb{R})\right)$.

(e) For $\omega_{n, c} \in \mathcal{S}_{\text {swn }}$ we have the following equivalences:

(i) $n(n+1)>|c|^{2}$;

(ii) $\omega_{n, c}$ corresponds to a point in the interior $\tilde{B}_{1}^{o}$;

(iii) $\omega_{n, c}$ is a faithful state on the von Neumann algebra $\Pi_{n, c}$ $\left(\mathcal{W}\left(L^{2}(\mathbb{R})\right)\right)^{\prime \prime}$.

Proof. The geometric characterizations within the half ball $\tilde{B}_{1}$ are immediate with Theorems 2.1 and 2.3 .

(b): For the gauge transformations $\nu_{z}, z \in \mathcal{T}$ it holds $\left\langle\nu_{z}\left(\omega_{n, c}\right) ; W(f)\right\rangle=$ $\left\langle\omega_{n, c} ; W(z f)\right\rangle=\exp \left\{-(1 / 4) s_{n, c}(z f, z f)\right\}$. But with equation (2.10) we have $s_{n, c}(z f, z f)=s_{n, c}(f, f) \forall z \in \mathcal{T}$, if and only if $c=0$. For the rest, see [30], [45].

(d): Let $s, \theta$ and $\kappa$ as in Theorem 2.3. Since $\nu_{s, \theta}$ is an affine bijection on $\mathcal{S}$, it follows from $\nu_{s, \theta}\left(\omega_{\kappa}\right)=\omega_{n, c}$ (Equation (2.19)), that $\omega_{n, c}$ is pure, if and only if $\omega_{\kappa}$ is pure. But by [30], [45] $\omega_{\kappa}$ is pure, if and only if $\kappa=0$, which with $(2.22)$ is equivalent to $n(n+1)=|c|^{2}$.

(e): Let $\left(\Pi_{\kappa}, \mathcal{H}_{\kappa}, \Omega_{\kappa}\right)$ be the GNS representation of the state $\omega_{\kappa} \in \mathcal{S}_{\text {temp }}$. Then Equation (2.19) yields $\left(\Pi_{\kappa} \circ \alpha_{s, \theta}, \mathcal{H}_{\kappa}, \Omega_{\kappa}\right)$ to be the GNS representation of $\omega_{n, c}$. Consequently, $\Pi_{\kappa}\left(\mathcal{W}\left(L^{2}(\mathbb{R})\right)\right)^{\prime \prime}=\Pi_{n, c}\left(\mathcal{W}\left(L^{2}(\mathbb{R})\right)\right)^{\prime \prime}$ and $\Omega_{\kappa}=\Omega_{n, c}$, which ensures that $\omega_{n, c}$ is faithful, if and only if $\omega_{\kappa}$ is so. But by [30] $\omega_{\kappa}$ is faithful, if and only if $\kappa \neq 0$, which with $(2.22)$ is equivalent to $n(n+1)>$ $|c|^{2}$.

\section{§2.4. Classicality and non-classicality}

An $\omega \in \mathcal{S}$ is defined to be a classical state on $\mathcal{W}\left(L^{2}(\mathbb{R})\right)$, if and only if the 
mapping

$$
L^{2}(\mathbb{R}) \ni f \longmapsto\langle\omega ; W(f)\rangle \exp \left\{\frac{1}{4}\|f\|^{2}\right\}
$$

is a positive-definite function on the additive group $L^{2}(\mathbb{R})$ [22], [15]. This definition is a generalization of the notion of a positive $P$-representation usually given in quantum optics. Let us denote by $\mathcal{S}_{\mathrm{cl}}$ the classical states on $\mathcal{W}\left(L^{2}(\mathbb{R})\right)$, and thus the complement $\mathcal{S}_{\text {non-cl }}=\mathcal{S} \backslash \mathcal{S}_{\text {cl }}$ consists of all non-classical states. For more details we refer to [46], [26], and [27].

Let us now consider the (squeezed) white noise states $\mathcal{S}_{\text {swn }}$ from Theorem 2.1.

Theorem 2.6. Let $n \geq 0$ and $c \in \mathbb{C}$ with $n(n+1) \geq|c|^{2}$. Then the (squeezed) white noise state $\omega_{n, c}$ is a classical state, if and only if $n \geq|c|$.

Especially, every temperature state is classical, that is,

$$
\mathcal{S}_{\text {temp }} \subset \mathcal{S}_{\text {cl }} \text {. }
$$

Furthermore, the set of all non-classical squeezed white noise states is given by

$$
\mathcal{S}_{\text {swn }} \bigcap \mathcal{S}_{\text {non }-\mathrm{cl}}=\left\{\omega_{n, c} \in \mathcal{S}_{\text {swn }}\left|0 \neq c \in \mathbb{C}, \sqrt{|c|^{2}+1 / 4}-1 / 2 \leq n<\right| c \mid\right\} .
$$

Proof. From the equations (2.10) and (2.11) it follows that

$$
\begin{aligned}
P_{n, c}(f):=\left\langle\omega_{n, c} ; W(f)\right\rangle \exp \left\{\frac{1}{4}\|f\|^{2}\right\} & =\exp \left\{-\frac{1}{2}\left(n\|f\|^{2}+\operatorname{Re}(c\langle f \mid J f\rangle)\right)\right\} \\
\forall f & \in L^{2}(\mathbb{R}) .
\end{aligned}
$$

Obviously, $P_{n, c}$ is a positive-definite function, if and only if $n\|f\|^{2}$ $+\operatorname{Re}(c\langle f \mid J f\rangle) \geq 0 \forall f \in L^{2}(\mathbb{R})$. This finally yields the result.

Let us turn to a characterization of the classicality of the (squeezed) white noise states in terms of their fluctuations compared with those of the vacuum. Because of the specific form of the states $\omega_{n, c}$ the results from [27, Section 3.4] reduce to the following.

Corollary 2.7. The state $\omega_{n, c} \in \mathcal{S}_{\mathrm{swn}}$ is classical, if and only if its MinVar-fluctuations are larger than or equal to the vacuum fluctuations. More precisely, we have the following equivalences:

(i) $\omega_{n, c} \in \mathcal{S}_{\mathrm{cl}}$,

(ii) $\operatorname{Min} \operatorname{Var}\left(\omega_{n, c} ; t\right) \geq \operatorname{Min} \operatorname{Var}\left(\omega_{0} ; t\right)=\operatorname{Max} \operatorname{Var}\left(\omega_{0} ; t\right)=t / 2$ for some $t>0$, 
(iii) $2 \kappa \geq \exp \{2 s\}-1$, where $s$ and $\kappa$ are given in terms of $n$ and $c$ by the equations (2.20) and (2.22), so that (2.19) is valid,

(iv) $n \geq|c|$.

Proof. Immediate consequence of Proposition 2.4, [27, Proposition 3.11] and the above Theorem.

Let us now consider a fixed temperature state $\omega_{\kappa} \in \mathcal{S}_{\text {temp }}, \kappa \geq 0$, which is squeezed by $\nu_{s, \theta}$ with varying squeezing strength $s \geq 0$. Then from Proposition 2.4 it follows that for each $t>0$ the mapping

$$
\mathbb{R} \ni s \longmapsto \operatorname{Min} \operatorname{Var}\left(\nu_{s, \theta}\left(\omega_{\kappa}\right) ; t\right)=t\left(\kappa+\frac{1}{2}\right) \exp \{-2 s\}
$$

decreases because of the exponential term. Starting at $s=0$ with the classical state $\omega_{\kappa}$ the squeezed white noise states $\nu_{s, \theta}\left(\omega_{\kappa}\right)$ remain classical for all $s \in$ $[0,(1 / 2) \ln (2 \kappa+1)]$ according to Corollary 2.7. If the squeezing strength $s$ becomes larger than $(1 / 2) \ln (2 \kappa+1)$, then the squeezed states $\nu_{s, \theta}\left(\omega_{\kappa}\right)$ are rendered non-classical. If $\kappa=0$, that is starting with the vacuum state $\omega_{0}$, then the squeezed vacuum $\nu_{s, \theta}\left(\omega_{0}\right)$ is non-classical for every non-zero squeezing strength $s$.

\section{§2.5. A concrete GNS representation}

In the present subsection we give a concrete realization of the GNS representation for our (squeezed) white noise states $\mathcal{S}_{\text {swn }}$ from Theorem 2.1. In the following we identify each $f \in L^{2}(\mathbb{R})$ with the associated tupel $\vec{f}:=$ $(\operatorname{Re}(f), \operatorname{Im}(f))$ of the real Hilbert space $L^{2}\left(\mathbb{R}, \mathbb{R}^{2}\right)$. Then the symmetric realbilinear form $s_{n, c}$ on $L^{2}(\mathbb{R})$ from Equation (2.10) writes as $s_{n, c}(f, g)$ $=\left\langle\vec{f} \mid Q_{n, c} \vec{g}\right\rangle_{2}$ with the $2 \times 2$-matix

$$
Q_{n, c}:=2\left(\begin{array}{cc}
n+\frac{1}{2}+a & b \\
b & n+\frac{1}{2}-a
\end{array}\right),
$$

where $a:=\operatorname{Re}(c)$ and $b:=\operatorname{Im}(c)$, and $\langle\vec{f} \mid \vec{g}\rangle_{2}=\operatorname{Re}\langle f \mid g\rangle$ is the canonical scalar product on $L^{2}\left(\mathbb{R}, \mathbb{R}^{2}\right)$.

Now we introduce representations of the Araki-Woods-type [6], that is on the tensor product of two Fock spaces $\mathcal{F}_{+}\left(L^{2}(\mathbb{R})\right) \otimes \mathcal{F}_{+}\left(L^{2}(\mathbb{R})\right)$, by setting

$$
W_{L, M}(f):=W_{\mathcal{F}}(L \vec{f}) \otimes W_{\mathcal{F}}(M \vec{f}), \quad f \in L^{2}(\mathbb{R}),
$$

where $L, M \in M_{2}(\mathbb{R})$ are arbitrary for the moment $-M_{2}(\mathbb{R})$ denotes the real $2 \times 2$ matrices, and $W_{\mathcal{F}}(f)$ are the usual Weyl operators acting on the Fock 
space $\mathcal{F}_{+}\left(L^{2}(\mathbb{R})\right)$. By explicitly verifying the Weyl relations we receive at the following statement.

Lemma 2.8. There exists a unique representation $\Pi_{L, M}$ of $\mathcal{W}\left(L^{2}(\mathbb{R})\right)$ with $\Pi_{L, M}(W(f))=W_{L, M}(f) \forall f \in L^{2}(\mathbb{R})$, if and only if $\operatorname{det}(L)+\operatorname{det}(M)=1$.

Such Araki-Woods-like representations usually are taken for the realization of the GNS representation of quasifree factor states [53], [23], [24]. In the following we will do so for our (squeezed) white noise states $\mathcal{S}_{\text {swn }}$. By explicit computation we arrive at the following lemma, where $\Omega_{\mathcal{F}}$ is the Fock vacuum vector.

Lemma 2.9. It holds $\left\langle\omega_{n, c} ; W(f)\right\rangle=\left\langle\Omega_{\mathcal{F}} \otimes \Omega_{\mathcal{F}} \mid W_{L, M}(f) \Omega_{\mathcal{F}} \otimes \Omega_{\mathcal{F}}\right\rangle$ $\forall f \in L^{2}(\mathbb{R})$, if and only if $L^{*} L+M^{*} M=Q_{n, c}$.

According to Lemmas 2.8 and 2.9 we are now searching for $L, M \in M_{2}(\mathbb{R})$ such that both conditions $\operatorname{det}(L)+\operatorname{det}(M)=1$ and $L^{*} L+M^{*} M=Q_{n, c}$ are fullfilled, in order to get a representation of $\mathcal{W}\left(L^{2}(\mathbb{R})\right)$ on $\mathcal{F}_{+}\left(L^{2}(\mathbb{R})\right) \otimes \mathcal{F}_{+}\left(L^{2}(\mathbb{R})\right)$ where the state $\omega_{n, c}$ is realized as the vector state $\Omega_{\mathcal{F}} \otimes \Omega_{\mathcal{F}}$. Considering the isomorphism of $M_{2}(\mathbb{R})$ and $\mathbb{C}^{2}$, which identifies each $\left(\begin{array}{l}\lambda \tau \\ \mu \nu\end{array}\right) \in M_{2}(\mathbb{R})$ with $(\lambda+i \mu, \tau+i \nu) \in \mathbb{C}^{2}$, we obtain

Lemma 2.10. If we identify $L, M \in M_{2}(\mathbb{R})$ with the vectors $\psi, \phi \in \mathbb{C}^{2}$, then the equations $L^{*} L+M^{*} M=Q_{n, c}$ and $\operatorname{det}(L)+\operatorname{det}(M)=1$ are equivalent to

$$
\begin{aligned}
& \|\psi\|^{2}+\|\phi\|^{2}=4\left(n+\frac{1}{2}\right), \text { and } \\
& \left\langle\psi \mid \sigma_{1} \psi\right\rangle+\left\langle\phi \mid \sigma_{1} \phi\right\rangle=4 b, \\
& \left\langle\psi \mid \sigma_{2} \psi\right\rangle+\left\langle\phi \mid \sigma_{2} \phi\right\rangle=2, \\
& \left\langle\psi \mid \sigma_{3} \psi\right\rangle+\left\langle\phi \mid \sigma_{3} \phi\right\rangle=4 a,
\end{aligned}
$$

where $\sigma_{k}, k=1,2,3$, are the standard Pauli spin matrices.

The (squeezed) white noise state $\omega_{n, c}$ corresponds to the point $(1 /(n+$ $(1 / 2)))(b,(1 / 2), a) \in \tilde{B}_{1}$ by Theorem 2.5. Thus, according to Lemma 2.10 we are searching for vectors $\psi, \phi \in \mathbb{C}^{2}$ so that the convex combination of the points $\|\psi\|^{-2}\left(\left\langle\psi \mid \sigma_{1} \psi\right\rangle,\left\langle\psi \mid \sigma_{2} \psi\right\rangle,\left\langle\psi \mid \sigma_{3} \psi\right\rangle\right) \in S_{1}$ and $\|\phi\|^{-2}\left(\left\langle\phi \mid \sigma_{1} \phi\right\rangle,\left\langle\phi \mid \sigma_{2} \phi\right\rangle\right.$, $\left.\left\langle\phi \mid \sigma_{3} \phi\right\rangle\right) \in S_{1}$ with weights $\|\psi\|^{2} /(4(n+(1 / 2)))$ and $\|\phi\|^{2} /(4(n+(1 / 2)))$, respectively, represents the above point $(1 /(n+(1 / 2)))(b,(1 / 2), a)$ in order to construct a realization of the GNS representation for $\omega_{n, c}$ with the use of Lemmas 2.8 and 2.9 . 
As a natural choice we describe the point $(1 /(n+(1 / 2)))(b,(1 / 2), a) \in \tilde{B}_{1}$ as the convex combination of the opposite points $(1 / \rho)(b,(1 / 2), a)$ and $(1 / \rho)$ $(-b,-(1 / 2),-a)$ on the unit sphere $S_{1}$, where $\rho:=\sqrt{|c|^{2}+1 / 4}$. By identifying these vectors with the corresponding real $2 \times 2$-matrices we arrive at $(J$ is the above natural conjugation on $L^{2}(\mathbb{R})$ )

$$
\begin{aligned}
& L_{n, c}:=k_{n, c}^{L}\left(\begin{array}{rr}
\rho+a & b \\
0 & \frac{1}{2}
\end{array}\right)=k_{n, c}^{L}\left[\left(\frac{\rho}{2}+\frac{\bar{c}}{2}+\frac{1}{4}\right) \mathbb{1}+\left(\frac{\rho}{2}+\frac{c}{2}-\frac{1}{4}\right) J\right], \\
& M_{n, c}:=k_{n, c}^{M}\left(\begin{array}{cr}
\rho-a & -b \\
0 & -\frac{1}{2}
\end{array}\right)=k_{n, c}^{M}\left[\left(\frac{\rho}{2}-\frac{\bar{c}}{2}-\frac{1}{4}\right) \mathbb{1}+\left(\frac{\rho}{2}-\frac{c}{2}+\frac{1}{4}\right) J\right]
\end{aligned}
$$

with the constants $k_{n, c}^{L}:=\sqrt{(n+(1 / 2)+\rho) /(\rho(\rho+a))}$ and $k_{n, c}^{M}:=$ $\sqrt{(n+(1 / 2)-\rho) /(\rho(\rho-a))}$. Thus by construction it holds $\operatorname{det}\left(L_{n, c}\right)+$ $\operatorname{det}\left(M_{n, c}\right)=1$ and $L_{n, c}^{*} L_{n, c}+M_{n, c}^{*} M_{n, c}=Q_{n, c}$, which ensures by Lemma 2.9 that

$$
\left\langle\omega_{n, c} ; A\right\rangle=\left\langle\Omega_{\mathcal{F}} \otimes \Omega_{\mathcal{F}} \mid \Pi_{L_{n, c}, M_{n, c}}(A) \Omega_{\mathcal{F}} \otimes \Omega_{\mathcal{F}}\right\rangle \quad \forall A \in \mathcal{W}\left(L^{2}(\mathbb{R})\right),
$$

with the representation $\Pi_{L_{n, c}, M_{n, c}}$ of the $\mathrm{C}^{*}$-algebra $\mathcal{W}\left(L^{2}(\mathbb{R})\right)$ on the Hilbert space $\mathcal{F}_{+}\left(L^{2}(\mathbb{R})\right) \otimes \mathcal{F}_{+}\left(L^{2}(\mathbb{R})\right)$ from Lemma 2.8 .

To obtain a realization of the GNS representation of $\omega_{n, c}$ it remains to prove the cyclicity of $\Omega_{\mathcal{F}} \otimes \Omega_{\mathcal{F}}$, which is given for $n(n+1)>|c|^{2}$ by arguments from the literature [30], [23], [53], [24]. Observe that $M_{n, c}=0$, if and only if $n+(1 / 2)=\rho$, i.e., if and only if $n(n+1)=|c|^{2}$, or equivalently, $(1 /(n+$ $(1 / 2)))(b, 1 / 2, a) \in \tilde{S}_{1}$ lies on the unit sphere. In this case the vector $\Omega_{\mathcal{F}} \otimes$ $\Omega_{\mathcal{F}}$ is not cyclic for the representation $\Pi_{L_{n, c}, 0}$ of $\mathcal{W}\left(L^{2}(\mathbb{R})\right)$ from Lemma 2.8. Summarizing we have shown the following result.

Theorem 2.11. For $n \geq 0$ and $c \in \mathbb{C}$ with $n(n+1) \geq|c|^{2}$ let us consider the (squeezed) white noise state $\omega_{n, c} \in \mathcal{S}_{\mathrm{swn}}$. It holds:

(a) For $n(n+1)>|c|^{2}$, or equivalently for $(1 /(n+(1 / 2)))(b,(1 / 2), a) \in \tilde{B}_{1}^{o}$, a concrete realization of the GNS representation $\left(\Pi_{n, c}, \mathcal{H}_{n, c}, \Omega_{n, c}\right)$ for $\omega_{n, c}$ is given by

$\Pi_{n, c}:=\Pi_{L_{n, c}, M_{n, c}}, \quad \mathcal{H}_{n, c}:=\mathcal{F}_{+}\left(L^{2}(\mathbb{R})\right) \otimes \mathcal{F}_{+}\left(L^{2}(\mathbb{R})\right), \quad \Omega_{n, c}:=\Omega_{\mathcal{F}} \otimes \Omega_{\mathcal{F}}$.

For each $f \in L^{2}(\mathbb{R})$ the corresponding annihilation operator is given by

$$
\begin{aligned}
a_{n, c}(f)= & k_{n, c}^{L}\left[\left(\frac{\rho}{2}+\frac{c}{2}+\frac{1}{4}\right) a_{\mathcal{F}}(f) \otimes \mathbb{1}+\left(\frac{\rho}{2}+\frac{c}{2}-\frac{1}{4}\right) a_{\mathcal{F}}^{*}(J f) \otimes \mathbb{1}\right] \\
& +k_{n, c}^{M}\left[\left(\frac{\rho}{2}-\frac{c}{2}-\frac{1}{4}\right) \mathbb{1} \otimes a_{\mathcal{F}}(f)+\left(\frac{\rho}{2}-\frac{c}{2}+\frac{1}{4}\right) \mathbb{1} \otimes a_{\mathcal{F}}^{*}(J f)\right]
\end{aligned}
$$


(the creation operator $a_{n, c}^{*}(f)$ is the adjoint), where $a_{\mathcal{F}}(g)$ and $a_{\mathcal{F}}^{*}(g)$ are the usual annihilation and creation operators on the Fock space $\mathcal{F}_{+}\left(L^{2}(\mathbb{R})\right)$.

(b) For $n(n+1)=|c|^{2}$, or equivalently for $(1 /(n+(1 / 2)))(b,(1 / 2), a) \in \tilde{S}_{1}$, a concrete realization of the GNS representation $\left(\Pi_{n, c}, \mathcal{H}_{n, c}, \Omega_{n, c}\right)$ for $\omega_{n, c}$ is given by the modified Fock representation

$$
\Pi_{n, c}(W(f)):=W_{\mathcal{F}}\left(L_{n, c} \vec{f}\right) \forall f \in L^{2}(\mathbb{R}), \quad \mathcal{H}_{n, c}:=\mathcal{F}_{+}\left(L^{2}(\mathbb{R})\right), \quad \Omega_{n, c}:=\Omega_{\mathcal{F}} .
$$

\section{§3. Quantum Stochastic Calculus}

In the present Section we suppose to be given a fixed (squeezed) white noise state $\omega_{n, c}$, which corresponds to an element of the interior $\tilde{B}_{1}^{o}$, or equivalently, according to Theorem 2.5 (e) $\omega_{n, c} \in \mathcal{S}_{\text {swn }}$ normally extends to the faithful state $\langle\omega ; \cdot\rangle=\langle\Omega \mid \cdot \Omega\rangle$ on the von Neumann algebra $\mathcal{C} \equiv \mathcal{C}_{n, c}=\Pi_{n, c}\left(\mathcal{W}\left(L^{2}(\mathbb{R})\right)\right)^{\prime \prime}$, where $\Omega \equiv \Omega_{n, c}$ is the GNS cyclic vector of $\omega_{n, c}$ resp. of $\omega$. For notational convenience its GNS Hilbert space is here denoted by

$$
\mathcal{H}_{n, c}=: L^{2}(\mathcal{C}, \omega) .
$$

Note that the normal extension $\omega$ of $\omega_{n, c}$ from $\mathcal{W}\left(L^{2}(\mathbb{R})\right)$ to the von Neumann algebra $\mathcal{C}$ is also called (squeezed) white noise.

For each $t \in \mathbb{R}$ let us define the ${ }^{*}$-automorphism $\sigma_{t}$ on $\mathcal{C}$ as the $\Pi_{n, c}$-normal extension of the Bogoliubov transformation $\sigma_{t}(W(f))=W\left(s_{t} f\right), f \in L^{2}(\mathbb{R})$, with the unitary right shift $s_{t}$ on $L^{2}(\mathbb{R})$ given by $s_{t} f(x)=f(x-t)$ for all $x \in \mathbb{R}$. Observe that our (squeezed) white noise state $\omega_{n, c}$ is invariant with respect to the shift $\sigma_{t}$.

The quantum stochastic calculus for the (squeezed) white noise state $\omega_{n, c}$ on $\mathcal{W}\left(L^{2}(\mathbb{R})\right)$ resp. $\omega$ on $\mathcal{C}$ is now constructed along the lines of [19].

\section{§3.1. Stochastic independence and generalized white noise}

For an arbitrary interval $I \subseteq \mathbb{R}$ the Weyl algebra $\mathcal{W}\left(L^{2}(I)\right)$ over the subHilbert space $L^{2}(I) \subseteq L^{2}(\mathbb{R})$ is a $C^{*}$-subalgebra of $\mathcal{W}\left(L^{2}(\mathbb{R})\right)$. According to this fact let us introduce for every interval $I \subseteq \mathbb{R}$ (the single point set $\{s\} \equiv[s, s]$, $s \in \mathbb{R}$, being also considered as an interval) the sub-von Neumann algebra

$$
\mathcal{C}_{I}:=\Pi_{n, c}\left(\mathcal{W}\left(L^{2}(I)\right)\right)^{\prime \prime} \subseteq \mathcal{C} .
$$

We introduce the conditional expectation from $\mathcal{C}$ onto $\mathcal{C}_{I}$ by extending

$$
Q_{I}\left(\Pi_{n, c}(W(f))\right)=\exp \left\{-\frac{1}{4} s_{n, c}\left(f, f \cdot \chi_{\mathbb{R} \backslash I}\right)\right\} \Pi_{n, c}\left(W\left(f \cdot \chi_{I}\right)\right),
$$


where $\chi_{I}$ is the characteristic function of the interval $I$. Especially for $Q_{0} \equiv$ $Q_{[0,0]}$ it holds $Q_{0}(A)=\langle\omega ; A\rangle \mathbb{1}=\langle\Omega \mid A \Omega\rangle \mathbb{1}$ for all $A \in \mathcal{C}$.

We add from now on to the noise system another system, the observable algebra $\mathcal{A}_{0}$ of which being given by an arbitrary von Neumann algebra and $\psi$ being a faithful normal reference state on $\mathcal{A}_{0}$. The (noncommutative or quantum) probability space $\left(\mathcal{A}_{0}, \psi\right)$ represents a physical system to which we want to couple our (squeezed) white noise system (for the notions of quantum probability, see [1], [34], [37], also [19]). Let us define the following tensor products to describe the uncoupled composite system (id means the identity mapping)

$$
\begin{aligned}
\mathcal{A} & :=\mathcal{A}_{0} \otimes \mathcal{C}, \\
\mathcal{A}_{I} & :=\mathcal{A}_{0} \otimes \mathcal{C}_{I}, \\
S_{t} & :=\mathrm{id} \otimes \sigma_{t}, \\
\varphi & :=\psi \otimes \omega, \\
P_{0} & :=\mathrm{id} \otimes Q_{0}, \\
P_{I} & :=\mathrm{id} \otimes Q_{I} .
\end{aligned}
$$

The GNS cyclic vector for the tensor product state $\varphi=\psi \otimes \omega$ is given by $\Omega_{\varphi}=$ $\Omega_{\psi} \otimes \Omega$, which is contained in the GNS Hilbert space $L^{2}(\mathcal{A}, \varphi)=L^{2}\left(\mathcal{A}_{0}, \psi\right) \otimes$ $L^{2}(\mathcal{C}, \omega)$.

Within this tensor product construction we identify $\mathcal{A}_{0} \equiv \mathcal{A}_{[0,0]}$ with $\mathcal{A}_{0} \otimes$ 1. $\varphi$ is a faithful normal state on the von Neumann algebra $\mathcal{A}$ and thus the tuple $(\mathcal{A}, \varphi)$ constitutes again a quantum probability space.

Furthermore, it is immediately checked that the triple $\left(\mathcal{A}, \varphi, S_{t}\right)$ represents a stationary dynamical system, and $\left(\mathcal{A}_{I}\right)_{I}$ is a filtration of the probability space $(\mathcal{A}, \varphi)$ with the associated conditional expectations $\left(P_{I}\right)_{I}$ (i.e., each $P_{I}$ is a completely positive operator on $\mathcal{A}$ with range $\mathcal{A}_{I}$ so that $\varphi \circ P_{I}=\varphi, P_{I}(\mathbb{1})=\mathbb{1}$ and $P_{I}^{2}=P_{I}$; cf. [51] Sections III.3 and IV.3).

Calculations using the Weyl relations prove that the quadruple $\left(\mathcal{A}, \varphi, S_{t}\right.$; $\left.\left(\mathcal{A}_{I}\right)_{I}\right)$ is indeed a quantum or generalized white noise over the von Neumann algebra $\mathcal{A}_{0}$ in the sense of [35], [36], [19], that is, the stationary dynamical system $\left(\mathcal{A}, \varphi, S_{t}\right)$ satisfies

(a) $S_{t} \circ P_{0}=P_{0}$, and $S_{t}\left(\mathcal{A}_{I}\right)=\mathcal{A}_{I+t}$ for all $t \in \mathbb{R}$, where $I+t:=\{s+t \mid$ $s \in I\}$.

(b) For two intervals $I, J \subseteq \mathbb{R}$ the algebras $\mathcal{A}_{I}$ and $\mathcal{A}_{J}$ are independent over $\mathcal{A}_{0}$ in $(\mathcal{A}, \varphi)$, whenever $|I \cap J|=0$, i.e., we have $P_{0}(A B C)=$ $P_{0}\left(A P_{0}(B) C\right)$ for all $A, C \in \mathcal{A}_{I}$ and all $B \in \mathcal{A}_{J}(|I \cap J|$ means the Lebesgue measure of the intersection $I \cap J)$. 
Especially, taking the simplest case $\mathcal{A}_{0}=\mathbb{C}$ we obtain that our (squeezed) white noise system $\left(\mathcal{C}, \omega, \sigma_{t} ;\left(\mathcal{C}_{I}\right)_{I}\right)$ constitutes a generalized white noise over $\mathbb{C}$ in the mentioned sense of [35], [36], [19]. Clearly, $\left(\mathcal{A}, \varphi, S_{t} ;\left(\mathcal{A}_{I}\right)_{I}\right)$ is an amplification of $\left(\mathcal{C}, \omega, \sigma_{t} ;\left(\mathcal{C}_{I}\right)_{I}\right)$.

\section{§3.2. Couplings to (squeezed) white noise}

The *-automorphism group $S_{t}=\mathrm{id} \otimes \sigma_{t}, t \in \mathbb{R}$, constitutes a $\mathrm{W}^{*}$-dynamical system on the von Neumann algebra $\mathcal{A}=\mathcal{A}_{0} \otimes \mathcal{C}$, which may be regarded as the free evolution of the photon system of the (squeezed) white noise. Let $u \equiv\left(u_{t}\right)_{t \geq 0} \subset \mathcal{A}$ be a unitary cocycle with respect to $S_{t}$, that is, $t \mapsto u_{t}$ is weak ${ }^{*}$-continuous and satisfies the cocycle relation

$$
u_{s+t}=S_{t}\left(u_{s}\right) u_{t}, \quad \forall s, t \geq 0 .
$$

Defining the inner ${ }^{*}$-automorphisms $C_{t}():.=u_{t}^{*} \cdot u_{t}$ for $t \geq 0$ and $C_{t}:=S_{t} \circ$ $C_{-t}^{-1} \circ S_{-t}$ for $t<0$, we obtain from (3.2) the cocycle identity

$$
C_{s+t}=C_{s} \circ S_{s} \circ C_{t} \circ S_{-s}, \quad \forall s, t \in \mathbb{R} .
$$

In terms of these *-automorphisms we may construct an interaction (coupling) of the photon field with the system described by $\mathcal{A}_{0}$ by setting for the interacting dynamics

$$
T_{t}:=C_{t} \circ S_{t}, \quad \forall t \in \mathbb{R}
$$

By construction $T_{t}, t \in \mathbb{R}$, is a one-parameter group of *-automorphisms on $\mathcal{A}$ constituting the $\mathrm{W}^{*}$-dynamical system $\left(\mathcal{A}, T_{t}\right)$ of the interacting system. The corresponding interaction operator is derived in Subsection 3.6 in a formal manner.

\section{§3.3. Quantum markov processes}

Let us recall that the centralizer $\mathcal{A}^{\varphi}$ of the faithful normal state $\varphi$ on the von Neumann algebra $\mathcal{A}$ is defined by $\mathcal{A}^{\varphi}:=\{A \in \mathcal{A} \mid\langle\varphi ;[A, B]\rangle=0 \forall B \in \mathcal{A}\}$.

In order that the interacting dynamics $T_{t}$ of the previous Subsection constitutes a stationary quantum Markov processes $\left(\mathcal{A}, \varphi, T_{t} ; \mathcal{A}_{0}\right)$ with values in $\mathcal{A}_{0}$ [34], [35], expressing a coupling to the generalized white noise $\left(\mathcal{A}, \varphi, S_{t} ;\left(\mathcal{A}_{I}\right)_{I}\right)$, the unitary cocycle $u$ has to be adapted and contained in the centralizer $\mathcal{A}^{\varphi}$ of the non-commutative probability space $(\mathcal{A}, \varphi)$. That means $u_{t} \in \mathcal{A}_{[0, t]} \cap \mathcal{A}^{\varphi}$ for all $t \geq 0$ [19, Lemma 3.1]. Then each *automorphism $C_{t}$ leaves $\varphi$ invariant. 
The dynamics of the above constructed Markov process $\left(\mathcal{A}, \varphi, T_{t} ; \mathcal{A}_{0}\right)$ reduced to $\mathcal{A}_{0}$ is the $\mathrm{W}^{*}$-dynamical semigroup

$$
R_{t}:=P_{0} \circ T_{t} \circ P_{0}=\exp \left\{t G_{L}\right\}, \quad t \geq 0,
$$

giving the irreversible stationary dynamical system $\left(\mathcal{A}_{0}, \psi, R_{t}\right)$ [1], [34], [37], where $G_{L}$ denotes the Lindblad generator of the semigroup $\left(R_{t}\right)_{t \geq 0}$.

In the present Subsection we recapitulate some results from the general theory, developed in [19], concerning non-commutative stochastic processes. There it is constructed the totality of all adapted unitary cocycles $u \subset \mathcal{A}^{\varphi}$, that are those unitary cocycles, which lead to quantum Markov processes as couplings to generalized white noise. For mathematical convenience, however, we restrict in the following to the case of a finite dimensional $\mathcal{A}_{0}$.

We take the construction of the (right) Hilbert $\mathrm{W}^{*}$-module $L^{2}\left(\mathcal{A}, P_{0}\right)$ over $\mathcal{A}_{0}$ from [19], equipped with the $\mathcal{A}_{0}$-valued inner product $\langle A \mid B\rangle_{0}=P_{0}\left(A^{*} B\right)$, $A, B \in \mathcal{A}$ (and completion). Since $\mathcal{A}_{0}$ is finite dimensional, the Hilbert module $L^{2}\left(\mathcal{A}, P_{0}\right)$ is isomorphic to the GNS-Hilbert space $L^{2}(\mathcal{A}, \varphi)$ of $\varphi$ in the sense of Banach spaces - the isomorphism is simply given by the extension of the injective mapping $\mathcal{A} \ni A \mapsto A \Omega_{\varphi}$. It is well known that the GNS sub-Hilbert space $L^{2}\left(\mathcal{A}^{\varphi}, \varphi\right) \subseteq L^{2}(\mathcal{A}, \varphi)$ of $\varphi$ restricted to the centralizer $\mathcal{A}^{\varphi}$ is given by a tracial non-commutative $L^{2}$-space [43], [52], that is, $L^{2}\left(\mathcal{A}^{\varphi}, \varphi\right)$ consists just of the closed operators $X$ affiliated to the centralizing von Neumann algebra $\mathcal{A}^{\varphi}$, such that the cyclic vector $\Omega_{\varphi}$ is contained in the domain of $X$. In this context $L^{4}\left(\mathcal{A}^{\varphi}, \varphi\right)$ is defined to consist of those closed $\mathcal{A}^{\varphi}$-affiliated operators $X \in$ $L^{2}\left(\mathcal{A}^{\varphi}, \varphi\right)$ with $\Omega_{\varphi} \in \mathcal{D}\left(X^{*} X\right)$. Especially it holds $L^{4}\left(\mathcal{A}^{\varphi}, \varphi\right) \subseteq L^{2}\left(\mathcal{A}^{\varphi}, \varphi\right)$, and if $X \in L^{p}\left(\mathcal{A}^{\varphi}, \varphi\right)$, then $X^{*} \in L^{p}\left(\mathcal{A}^{\varphi}, \varphi\right), p=2,4$.

By means of the additive cocycles $\beta=\left(\beta_{t}\right)_{t \geq 0} \subset L^{2}\left(\mathcal{A}, P_{0}\right)$ with respect to the generalized white noise $\left(\mathcal{A}, \varphi, S_{t} ;\left(\mathcal{A}_{I}\right)_{I}\right)$ - satisfying the cocycle identity $\beta_{s+t}=\beta_{t}+S_{t}\left(\beta_{s}\right) \forall s, t \geq 0$, and the adaptedness $\beta_{t} \in L^{2}\left(\mathcal{A}_{[0, t]}, P_{0}\right) \forall t \geq 0-$ it has been constructed the left stochastic Itô-integral

$$
\int_{0}^{t} d \beta_{s} \cdot x_{s}, \quad t \geq 0,
$$

for an adapted stochastic process $x=\left(x_{s}\right)_{s \geq 0} \subset L^{2}\left(\mathcal{A}, P_{0}\right)$, which is continuous with respect to the $\|\cdot\|_{0}$-topology arising from $\langle\cdot \mid \cdot\rangle_{0}$. Recalling from [19] the definition of the mutual quadratic variation

$$
\llbracket \beta, \gamma \rrbracket_{t}:=\lim _{|\mathcal{Z}| \rightarrow 0} \sum_{j<n_{\mathcal{Z}}}\left(\beta_{t_{j+1}}-\beta_{t_{j}}\right)\left(\gamma_{t_{j+1}}-\gamma_{t_{j}}\right)
$$

for two additive cocycles $\beta$ and $\gamma$ in $L^{4}\left(\mathcal{A}^{\varphi}, \varphi\right)$, where $|\mathcal{Z}|$ denotes the mesh of 
the partition $\mathcal{Z}:=\left\{t_{j} \mid 0=t_{0}<t_{1}<\cdots<t_{n_{\mathcal{Z}}}=t\right\}$ of the interval $[0, t]$, we communicate from [19] the following result.

Theorem 3.1. $\quad$ There is a one-to-one correspondence between

(i) $\|\cdot\|_{0}$-continuous adapted unitary cocycles $u \subset \mathcal{A}^{\varphi}$, and

(ii) additive cocycles $\beta \subset L^{4}\left(\mathcal{A}^{\varphi}, \varphi\right)$ with the mutual quadratic variation $\llbracket \beta^{*}, \beta \rrbracket_{t}=-\left(\beta_{t}^{*}+\beta_{t}\right)$, which is given in terms of the stochastic differential equation $d u_{t}=d \beta_{t} \cdot u_{t}$ with $u_{0}=\mathbb{1}$, i.e. by

$$
u_{t}=\mathbb{1}+\int_{0}^{t} d \beta_{s} \cdot u_{s}, \quad t \geq 0,
$$

if the additive cocycle $\beta$ is given, respectively by

$$
\beta_{t}=\lim _{n \rightarrow \infty} \sum_{j=0}^{n-1} S_{j t / n}\left(u_{t / n}-\mathbb{1}\right),
$$

whenever the unitary cocycle $u$ is given.

The above Theorem shows that quantum Markov processes, which arise from couplings to generalized white noise are given by adapted additive cocycles. An additive cocycle is considered as a quantum Brownian motion.

Suppose now the unitary cocycle $u$ to be constructed in terms of the additive cocycle $\beta \subset L^{4}\left(\mathcal{A}^{\varphi}, \varphi\right) \subseteq L^{2}(\mathcal{A}, \varphi) \cong L^{2}\left(\mathcal{A}, P_{0}\right)$ from (ii). Then the interacting dynamics $T_{t}$ from (3.4) constitutes the associated stationary Markov process $\left(\mathcal{A}, \varphi, T_{t} ; \mathcal{A}_{0}\right)$ with values in $\mathcal{A}_{0}$. The reduced dynamics $\left(R_{t}\right)_{t \geq 0}$ from Equation (3.5) is given by

$$
R_{t}(A)=P_{0}\left(T_{t}(A)\right)=\exp \left\{t G_{L}\right\}(A)=\left\langle u_{t} \mid A u_{t}\right\rangle_{0}, \quad A \in \mathcal{A}_{0},
$$

with the Lindblad generator

$$
G_{L}(A)=\left\langle b_{1} \mid A b_{1}\right\rangle_{0}+K^{*} A+A K, \quad A \in \mathcal{A}_{0},
$$

where $K:=P_{0}\left(\beta_{1}\right)$ and $b_{t}:=\beta_{t}-K t$. If $b_{t}^{*}=-b_{t}$, then the $\mathrm{W}^{*}$-dynamical semigroup $R_{t}=\exp \left\{t G_{L}\right\}$ obeys detailed balance (in the sense of [33]).

For practical purposes it is not necessary to verify the demand $\llbracket \beta^{*}, \beta \rrbracket_{t}=$ $-\left(\beta_{t}^{*}+\beta_{t}\right)$ for the quadratic variation from part (ii) of the above Theorem. The following result from [19] ensures some equivalent, but easier treatable conditions.

Proposition 3.2. $\quad$ Introducing for the adapted additive cocycle $\beta \subset L^{4}$ $\left(\mathcal{A}^{\varphi}, \varphi\right)$ the quantities $K:=P_{0}\left(\beta_{1}\right)$, and $b_{t}:=\beta_{t}-K t \in L^{4}\left(\mathcal{A}^{\varphi}, \varphi\right)\left(b_{t}\right.$ turns 
out to be a centered additive cocycle), and $F(t):=\left\|b_{t}^{*} b_{t} \Omega_{\varphi}\right\|^{2}$, then the condition $\llbracket \beta^{*}, \beta \rrbracket_{t}=-\left(\beta_{t}^{*}+\beta_{t}\right)$ for the mutual quadratic variation of part (ii) of Theorem 3.1 is equivalent to the following three conditions

(a) $P_{0}\left(b_{t}^{*} b_{t}\right)=-\left(K^{*}+K\right) t$;

(b) $\left\|\left(b_{t}^{*}+b_{t}\right) \Omega_{\varphi}\right\|^{2}=t((d F) /(d t))(0)$;

(c) $\left\|\left(b_{t}^{*}+b_{t}\right) \Omega_{\varphi}\right\|^{2}=\left\langle b_{t}^{*} b_{t} \Omega_{\varphi} \mid\left(b_{t}^{*}+b_{t}\right) \Omega_{\varphi}\right\rangle$.

\section{§3.4. Canonical additive cocycles}

In the present Subsection we construct by use of the annihilation and creation operators of our (squeezed) white noise state $\omega_{n, c}$ adapted additive cocycles $\beta$, which fulfill the conditions of Theorem 3.1. Considering the interacting cocycle dynamics $T_{t}()=.u_{t}^{*} S_{t}(.) u_{t}$ of the associated Markov processes, we give the corresponding Lindblad generators $G_{L}$ of the reduced dynamics $R_{t}$ from the Eqs. (3.7) and (3.8). The derivation in a formal sense of the associated interaction operators $\Gamma$ is defered to Subsection 3.6.

The tensor product construction from Subsection 3.1 suggests the following ansatz for an adapted (centered) additive cocycle

$$
b_{t}:=M_{1} \otimes C(t)+M_{2} \otimes C^{*}(t), \quad t \geq 0,
$$

with some closed operators $C(t)$ affiliated to $\mathcal{C}_{[0, t]}$ fulfilling the cocycle relations $C(s+t)=C(t)+\sigma_{t}(C(s))$, and with $M_{1}, M_{2} \in \mathcal{A}_{0}$.

In order that $b_{t}$ fulfills part (ii) of Theorem 3.1 it has to be affiliated to the centralizer $\mathcal{A}^{\varphi}$. The centralizer $\mathcal{A}^{\varphi}$ consists just of the fixed points for the modular group $\Sigma_{t}^{\varphi}$ associated with the faithful normal state $\varphi[44$, Lemma 8.14.6], that is, we have

$$
\mathcal{A}^{\varphi}=\{A \in \mathcal{A} \mid\langle\varphi ;[A, B]\rangle=0 \forall B \in \mathcal{A}\}=\left\{A \in \mathcal{A} \mid \Sigma_{t}^{\varphi}(A)=A\right\} .
$$

According to the tensor product structure $\varphi=\psi \otimes \omega$ the modular group $\Sigma_{t}^{\varphi}$ decomposes into the tensor product $\Sigma_{t}^{\varphi}=\Sigma_{t}^{\psi} \otimes \Sigma_{t}^{\omega}$ of the modular groups of $\psi$ resp. $\omega$. Consequently, in order that $b_{t}$ is affiliated to the centralizer $\mathcal{A}^{\varphi}$, it is not necessary that $M_{1}, M_{2}$ are contained in the centralizer $\mathcal{A}_{0}^{\psi}$ of $\psi$ and that $C(t)$ is affiliated to the centralizer $\mathcal{C}^{\omega}$ of $\omega$. E.g., suppose $C(t)$ to be an eigenelement of the modular group $\Sigma_{t}^{\omega}$ with $\Sigma_{t}^{\omega}(C(t))=c^{-i t} C(t)$ for all $t \in \mathbb{R}$ and a $c \geq 0$, then we have that $b_{t}$ is affiliated to $\mathcal{A}^{\varphi}$, if $M_{1}, M_{2}$ are eigenelements of the modular group $\Sigma_{t}^{\psi}$ with $\Sigma_{t}^{\psi}\left(M_{1}\right)=c^{i t} M_{1}$ and $\Sigma_{t}^{\psi}\left(M_{2}\right)=c^{-i t} M_{2}$. This means that in the case of a non-commutative $\mathcal{A}_{0}$ with $\operatorname{dim}\left(\mathcal{A}_{0}\right) \geq 2$ the centralizer $\mathcal{A}^{\varphi}$ of $\varphi$ is larger that the tensor product $\mathcal{A}_{0}^{\psi} \otimes \mathcal{C}^{\omega}$ of the centralizers of $\psi$ and $\omega$. 
Let us make the ansatz $\beta_{t}=b_{t}+K t$ for the canonical additive cocycle, where the centered part $b_{t}$ is of the above type with the operator $C(t)$ taken as the annihilation operator $a_{n, c}(t):=a_{n, c}\left(\chi_{[0, t]}\right)$ in the representation of our (squeezed) white noise state $\omega_{n, c}$, smeared with the characteristic function $\chi_{[0, t]}$ of the interval $[0, t]$ for each $t \geq 0$. That is

$$
b_{t}:=M_{1} \otimes a_{n, c}(t)+M_{2} \otimes a_{n, c}^{*}(t), \quad t \geq 0,
$$

with $M_{1}, M_{2} \in \mathcal{A}_{0}$.

Now the additive cocycle $\beta_{t}$ is to be introduced in such way, that it satisfies the conditions of Theorem 3.1 resp. Proposition 3.2. First of all we have to ensure that $b_{t} \in L^{4}\left(\mathcal{A}^{\varphi}, \varphi\right)$. Since the considered state $\omega_{n, c}$ is entire analytic (cf. Subsection 2.1), it follows that $\Omega$ is contained in the domain of every polynomial of the annihilation and creation operators of $\omega_{n, c}$. Consequently, we have $\Omega_{\varphi} \in \mathcal{D}\left(b_{t}^{*} b_{t}\right)$. Since the annihilation operators $a_{n, c}(t)$ in general are not eigenelements of the modular group $\Sigma_{t}^{\omega}$, it is not easily seen from (3.9) under which circumstances $b_{t}$ is affiliated to $\mathcal{A}^{\varphi}$. We have to rewrite $b_{t}$ in a form easier to handle.

According to Theorem 2.3 the (squeezed) white noise state $\omega_{n, c}$ on $\mathcal{W}\left(L^{2}(\mathbb{R})\right)$ is given as the Bogoliubov transform of a unique white noise (temperature) state $\omega_{\kappa}$ by Equation (2.19),

$$
\nu_{s, \theta}\left(\omega_{\kappa}\right)=\omega_{n, c} .
$$

The GNS representations of $\omega_{n, c}$ and $\omega_{\kappa}$ are connected by the Bogoliubov transformation $\alpha_{s, \theta}$ on $\mathcal{W}\left(L^{2}(\mathbb{R})\right)$ arising from the symplectic transformation $T_{s, \theta}=\cosh (s) \mathbb{1}+\exp \{i \theta\} \sinh (s) J$ from Equation (2.16), that is, both GNS representations are on the same Hilbert space $L^{2}(\mathcal{C}, \omega)$ and have the same cyclic vector $\Omega$, but the representation morphisms are connected by

$$
\Pi_{n, c}=\Pi_{\kappa} \circ \alpha_{s, \theta},
$$

leading to disjoint representations. An immediate consequence is the fact that the associated field, annihilation resp. creation operators for the two states $\omega_{n, c}$ and $\omega_{\kappa}$ act on the same Hilbert space $L^{2}(\mathcal{C}, \omega)$ : If $a_{\kappa}(f)$ are the annihilation operators associated with the state $\omega_{\kappa}$, then we have the relation (cf. [25])

$$
a_{n, c}(f)=\cosh (s) a_{\kappa}(f)+\mathrm{e}^{i \theta} \sinh (s) a_{\kappa}^{*}(J f), \quad \forall f \in L^{2}(\mathbb{R}) .
$$

Proposition 3.3. Let $\Delta$ be the modular operator for the pair $(\mathcal{C}, \Omega)$ with the cyclic and separating vector $\Omega$ for the von Neumann algebra $\mathcal{C}($ e.g., 
$[12$, Section 2.5.2]). Then we have for all $t \in \mathbb{R}$ that

$$
\begin{aligned}
\Delta^{i t} \Pi_{\kappa}(A) \Omega & =\Pi_{\kappa}\left(\sigma_{t}^{\kappa}(A)\right) \Omega, \\
\Delta^{i t} \Pi_{n, c}(A) \Omega & =\Pi_{n, c}\left(\sigma_{t}^{n, c}(A)\right) \Omega, \\
\forall A & \in \mathcal{W}\left(L^{2}(\mathbb{R})\right),
\end{aligned}
$$

where $\sigma_{t}^{\kappa}$ and $\sigma_{t}^{n, c}$ are the Bogoliubov transformations on $\mathcal{W}\left(L^{2}(\mathbb{R})\right)$ given by

$$
\sigma_{t}^{\kappa}(W(f))=W\left(\gamma^{i t} f\right), \quad f \in L^{2}(\mathbb{R}),
$$

with $\gamma:=(1+\kappa) / \kappa$, and $\sigma_{t}^{n, c}=\alpha_{s, \theta}^{-1} \circ \sigma_{t}^{\kappa} \circ \alpha_{s, \theta}$. Especially it follows for the modular group $\Sigma_{t}^{\omega}()=.\Delta^{i t} . \Delta^{-i t}$ that

$$
\Sigma_{t}^{\omega}\left(a_{\kappa}^{*}(f)\right)=a_{\kappa}^{*}\left(\gamma^{i t} f\right)=\gamma^{i t} a_{\kappa}^{*}(f), \quad \forall f \in L^{2}(\mathbb{R}),
$$

that is, the creation resp. annihilation operators associated with $\omega_{\kappa}$ are elements of the eigenspace of the modular group $\Sigma_{t}^{\omega}$ with the eigenvalues $\gamma^{i t}$ resp. $\gamma^{-i t}$.

Proof. For $h_{1}, h_{2} \in L^{2}(\mathbb{R})$ we obtain with the Weyl relations (2.6) and with (2.18) that

$$
\begin{aligned}
& \left\langle\Pi_{\kappa}\left(W\left(h_{1}\right)\right) \Omega \mid \Pi_{\kappa}\left(W\left(h_{2}\right)\right) \Omega\right\rangle \\
& \quad=\exp \left\{\frac{i}{2} \operatorname{Im}\left\langle h_{1} \mid h_{2}\right\rangle\right\}\left\langle\omega_{\kappa} ; W\left(h_{2}-h_{1}\right)\right\rangle \\
& =\exp \left\{\frac{i}{2} \operatorname{Im}\left\langle h_{1} \mid h_{2}\right\rangle-\frac{2 \kappa+1}{4}\left\|h_{2}-h_{1}\right\|^{2}\right\} \\
& \quad=\exp \left\{\frac{1}{2(\gamma-1)}\left\langle h_{1} \mid h_{2}\right\rangle+\frac{\gamma}{2(\gamma-1)}\left\langle h_{2} \mid h_{1}\right\rangle-\frac{2 \kappa+1}{4}\left(\left\|h_{1}\right\|^{2}+\left\|h_{2}\right\|^{2}\right)\right\}
\end{aligned}
$$

where $\kappa=1 /(\gamma-1)$. Thus it follows for $f, g \in L^{2}(\mathbb{R})$ and every $t \in \mathbb{R}$ that

$$
\begin{aligned}
V_{f, g}(t) & :=\left\langle\Pi_{\kappa}(W(f)) \Omega \mid \Pi_{\kappa}\left(W\left(\gamma^{i t} g\right)\right) \Omega\right\rangle \\
& =\exp \left\{\frac{1}{2(\gamma-1)} \gamma^{i t}\langle f \mid g\rangle+\frac{\gamma}{2(\gamma-1)} \gamma^{-i t}\langle g \mid f\rangle-\frac{2 \kappa+1}{4}\left(\|f\|^{2}+\|g\|^{2}\right)\right\},
\end{aligned}
$$

which extends uniquely to an entire holomorphic function on $\mathbb{C}$. Especially we have for every $t \in \mathbb{R}$ that

$$
\begin{aligned}
V_{f, g}(t-i) & =\exp \left\{\frac{1}{2(\gamma-1)}\left\langle\gamma^{i t} g \mid f\right\rangle+\frac{\gamma}{2(\gamma-1)}\left\langle f \mid \gamma^{i t} g\right\rangle-\frac{2 \kappa+1}{4}\left(\|g\|^{2}+\|f\|^{2}\right)\right\} \\
& =\left\langle\Pi_{\kappa}\left(W\left(\gamma^{i t} g\right)\right) \Omega \mid \Pi_{\kappa}(W(f)) \Omega\right\rangle .
\end{aligned}
$$

Since the linear hull of the represented Weyl operators $\left\{\Pi_{\kappa}(W(h)) \mid h \in L^{2}(\mathbb{R})\right\}$ is dense in $\mathcal{C}$ in the $\sigma$-weak topology, it follows from [32, Lemma 9.2.17] that

$$
\Sigma_{t}^{\omega}\left(\Pi_{\kappa}(A)\right)=\Delta^{i t} \Pi_{\kappa}(A) \Delta^{-i t}=\Pi_{\kappa}\left(\sigma_{t}^{\kappa}(A)\right), \quad \forall A \in \mathcal{W}\left(L^{2}(\mathbb{R})\right) .
$$


Especially the normal extension $\langle\omega ;\rangle=.\langle\Omega \mid . \Omega\rangle$ of $\omega_{\kappa}$ is a KMS state on $\mathcal{C}$ with respect to the $\mathrm{W}^{*}$-dynamics arising from the normal extension of the Bogoliubov automorphism group $\sigma_{t}^{\kappa}$. The connection of $\Pi_{\kappa}$ with $\Pi_{n, c}$ from Equation (3.11) immediately yields $\Sigma_{t}^{\omega}\left(\Pi_{n, c}(A)\right)=\Pi_{n, c}\left(\sigma_{t}^{n, c}(A)\right)$ for all $A \in$ $\mathcal{W}\left(L^{2}(\mathbb{R})\right)$.

By differentiation (3.14) extends to the field operators $\Sigma_{t}^{\omega}\left(\Phi_{\kappa}(f)\right)$ $=\Phi_{\kappa}\left(\gamma^{i t} f\right)$ for all $f \in L^{2}(\mathbb{R})$ and every $t \in \mathbb{R}$, which finally leads to (3.13).

Because of (3.12) the creation resp. annihilation operators associated with $\omega_{n, c}$ are not contained in the eigenspaces of the modular group $\Sigma_{t}^{\omega}$, provided $c \neq 0$ and hence $\omega_{n, c}$ is a proper squeezed white noise state.

The annihilation operators of $\omega_{\kappa}$ are eigenelements of $\Sigma_{t}^{\omega}$, and thus we have to transfrom $b_{t}$ with Equation (3.12). With $a_{\kappa}(t):=a_{\kappa}\left(\chi_{[0, t]}\right)$ we obtain

$$
b_{t}=L_{1} \otimes a_{\kappa}(t)+L_{2} \otimes a_{\kappa}^{*}(t), \quad t \geq 0,
$$

where the operators $M_{1}, M_{2} \in \mathcal{A}_{0}$ are connected with the $L_{1}, L_{2} \in \mathcal{A}_{0}$ according to

$$
M_{1}=\cosh (s) L_{1}-\mathrm{e}^{-i \theta} \sinh (s) L_{2}, \quad M_{2}=\cosh (s) L_{2}-\mathrm{e}^{i \theta} \sinh (s) L_{1},
$$

respectively,

$$
L_{1}=\cosh (s) M_{1}+\mathrm{e}^{-i \theta} \sinh (s) M_{2}, \quad L_{2}=\cosh (s) M_{2}+\mathrm{e}^{i \theta} \sinh (s) M_{1} .
$$

Since $a_{\kappa}(t)$ resp. $a_{\kappa}^{*}(t)$ are contained in the eigenspace of the modular group $\Sigma_{t}^{\omega}$ with the eigenvalue $\gamma^{-i t}$ resp. $\gamma^{i t}$, it follows that $b_{t}$ is affiliated to the centralizer $\mathcal{A}^{\varphi}$, if and only if $L_{1}$ resp. $L_{2}$ are contained in the eigenspace of the modular group $\Sigma_{t}^{\psi}$ associated with the faithful normal state $\psi$ on $\mathcal{A}_{0}$ with the eigenvalues $\gamma^{i t}$ resp. $\gamma^{-i t}$. As mentioned before, the latter property is only possible, if the dimension of our non-commutative $\mathcal{A}_{0}$ is larger than or equal to 2 , ensuring that $b_{t}$ can only be affiliated to the centralizer $\mathcal{A}^{\varphi}$ for $\operatorname{dim}\left(\mathcal{A}_{0}\right) \geq 2$. Note that the state $\psi$ on $\mathcal{A}_{0}$ is rather unspecified, which up to now allows for arbitrary $L_{1}, L_{2} \in \mathcal{A}_{0}$. Together with the domain condition $\Omega_{\varphi} \in \mathcal{D}\left(b_{t}^{*} b_{t}\right)$ we now have established that $b_{t} \in L^{4}\left(\mathcal{A}^{\varphi}, \varphi\right)$.

In order that the total additive cocycle $\beta_{t}=b_{t}+K t \in L^{4}\left(\mathcal{A}^{\varphi}, \varphi\right)$ with some suitable $K \in \mathcal{A}_{0}$ possesses a mutual quadratic variation of the form $\llbracket \beta^{*}, \beta \rrbracket_{t}=-\left(\beta_{t}^{*}+\beta_{t}\right)$, given in part (ii) of Theorem 3.1, we have to demand the three conditions in Proposition 3.2. Let us first calculate the function

$$
F(t)=\left\|b_{t}^{*} b_{t} \Omega_{\varphi}\right\|^{2}=\left\langle\varphi ; b_{t}^{*} b_{t} b_{t}^{*} b_{t}\right\rangle=\left\langle\psi \otimes \omega_{n, c} ; b_{t}^{*} b_{t} b_{t}^{*} b_{t}\right\rangle .
$$


Since $\omega_{n, c}$ is quasifree and satisfies the expectations (2.1) to (2.5) it is immediate to see that $F(t)$ is proportional to $t^{2}$, which implies $((d F) /(d t))(0)=0$. Condition (b) in Proposition 3.2 now implies $b_{t}=-b_{t}^{*}$, and thus we conclude that we have to demand $L_{2}^{*}=-L_{1}=: L$. Especially it follows that condition (c) is automatically fulfilled.

Summarizing, our ansatz (3.9) implies the centered part $b_{t}$ of our additive cocycle $\beta_{t}=b_{t}+K t$ to be

$$
b_{t}=M^{*} \otimes a_{n, c}(t)-M \otimes a_{n, c}^{*}(t)=L^{*} \otimes a_{\kappa}(t)-L \otimes a_{\kappa}^{*}(t),
$$

where the connection of the arbitrary choosable $M \in \mathcal{A}_{0}$ with $L \in \mathcal{A}_{0}$ (note that $\psi$ has to be chosen in accordance with $M$ resp. $L$ ) is given by

$$
M=\cosh (s) L+\mathrm{e}^{-i \theta} \sinh (s) L^{*}, \quad L=\cosh (s) M-\mathrm{e}^{-i \theta} \sinh (s) M^{*},
$$

for arbitrary $L \in \mathcal{A}_{0}$.

Condition (a) determines only the real part of $K \in \mathcal{A}_{0}$. Hence we put

$$
K:=-\frac{1}{2} P_{0}\left(b_{1}^{*} b_{1}\right)+i H
$$

with some arbitrarily choosable selfadjoint $H \in \mathcal{A}_{0}$.

Now let us consider the interacting dynamics $T_{t}$, i.e. we consider the stationary Markov process $\left(\mathcal{A}, \varphi, T_{t} ; \mathcal{A}_{0}\right)$ with values in $\mathcal{A}_{0}$, which is associated with the additive cocycle $\beta_{t}=b_{t}+K t$ from above (where $b_{t}$ is from (3.15) and $K$ from (3.17)) resp. with the unitary cocycle $u_{t}$ constructed from $\beta_{t}$ according to Equation (3.6). For the reduced dynamics $R_{t}=\exp \left\{t G_{L}\right\}$ on $\mathcal{A}_{0}$ from (3.7) or (3.5) we obtain the following result.

Proposition 3.4. The Lindblad generator $G_{L}$ from (3.8) has the form as

$$
\begin{aligned}
G_{L}(A)= & i[H, A]+(\kappa+1) L^{*} A L+\kappa L A L^{*} \\
& -\frac{\kappa+1}{2}\left(L^{*} L A+A L^{*} L\right)-\frac{\kappa}{2}\left(L L^{*} A+A L L^{*}\right), \quad A \in \mathcal{A}_{0},
\end{aligned}
$$

and obeys detailed balance (in the sense of [33]), since we here have $b_{t}^{*}=-b_{t}$. Here $L$ is connected with $M$ according to Equation (3.16).

Proof. According to (3.8) it holds $G_{L}(A)=P_{0}\left(b_{1}^{*} A b_{1}\right)+K^{*} A+A K$. But $P_{0}=\mathrm{id} \otimes Q_{0}$, where $Q_{0}(A)=\langle\omega ; A\rangle \mathbb{1}=\langle\Omega \mid A \Omega\rangle \mathbb{1}$ for all $A \in \mathcal{C}$. From (3.15) 
we have $b_{1}=M^{*} \otimes a_{n, c}(1)-M \otimes a_{n, c}^{*}(1)$. Thus we get

$$
\begin{aligned}
P_{0}\left(b_{1}^{*} A b_{1}\right)= & M A M^{*}\left\langle\omega_{n, c} ; a_{n, c}^{*}(1) a_{n, c}(1)\right\rangle+M^{*} A M\left\langle\omega_{n, c} ; a_{n, c}(1) a_{n, c}^{*}(1)\right\rangle \\
& -M A M\left\langle\omega_{n, c} ; a_{n, c}^{*}(1) a_{n, c}^{*}(1)\right\rangle-M^{*} A M^{*}\left\langle\omega_{n, c} ; a_{n, c}(1) a_{n, c}(1)\right\rangle \\
= & n M A M^{*}+(n+1) M^{*} A M-\bar{c} M A M-c M^{*} A M^{*},
\end{aligned}
$$

where we have used the evaluations from (2.2) to (2.5). Now the result is immediate with the relations connecting $n, c$ with $\kappa$ from Theorem 2.3.

\section{§3.5. The Itô table}

Let us here consider the additive cocycle $\beta_{t}=b_{t}+K t$, where $b_{t}$ is from (3.15) and $K$ from (3.17). $\beta_{t}$ has been constructed to satisfy the condition $\llbracket \beta^{*}, \beta \rrbracket_{t}=-\left(\beta_{t}^{*}+\beta_{t}\right)$, which is written in differentials as

$$
\begin{aligned}
d \beta_{t}^{*} d \beta_{t} & =d b_{t}^{*} d b_{t}=-d b_{t}^{*}-d b_{t}-\left(K^{*}+K\right) d t=-\left(K^{*}+K\right) d t \\
& =P_{0}\left(b_{1}^{*} b_{1}\right) d t=\left(n M M^{*}+(n+1) M^{*} M-\bar{c} M M-c M^{*} M^{*}\right) d t
\end{aligned}
$$

where we have used Equation (3.18), the relation $b_{t}^{*}=-b_{t}$ and the mutual quadratic variations $\llbracket \beta^{*}, t \rrbracket_{t}=\llbracket \beta, t \rrbracket_{t}=\llbracket t, t \rrbracket=0$. Especially we have

$$
d b_{t}^{*} d b_{t}=\left(n M M^{*}+(n+1) M^{*} M-\bar{c} M M-c M^{*} M^{*}\right) d t .
$$

On the other hand from Equation (3.15) we get formally

$$
d b_{t}=M^{*} \otimes d a_{n, c}(t)-M \otimes d a_{n, c}^{*}(t),
$$

which leads to the product formula

$$
\begin{aligned}
d b_{t}^{*} d b_{t}= & M M^{*} \otimes d a_{n, c}^{*}(t) d a_{n, c}(t)+M^{*} M \otimes d a_{n, c}(t) d a_{n, c}^{*}(t) \\
& -M M \otimes d a_{n, c}^{*}(t) d a_{n, c}^{*}(t)-M^{*} M^{*} \otimes d a_{n, c}(t) d a_{n, c}(t) .
\end{aligned}
$$

Comparison with Equation (3.19) gives the following Itô table:

\begin{tabular}{c|ccc}
$1 \backslash^{2}$ & $d a_{n, c}(t)$ & $d a_{n, c}^{*}(t)$ & $d t$ \\
\hline$d a_{n, c}(t)$ & $c d t$ & $(n+1) d t$ & 0 \\
$d a_{n, c}^{*}(t)$ & $n d t$ & $\bar{c} d t$ & 0 \\
$d t$ & 0 & 0 & 0
\end{tabular}


For example, we read off the table $d a_{n, c}(t) d a_{n, c}^{*}(t)=(n+1) d t$.

Note that $\beta_{t}$ is a well-defined additive cocycle satisfying the conditions in part (ii) of Theorem 3.1 only, if the dimension of $\mathcal{A}_{0}$ is equal to resp. larger than 2. Hence the above Itô table is valid only for composed systems $\mathcal{A}=\mathcal{A}_{0} \otimes \mathcal{C}$ with non-trivial $\mathcal{A}_{0}$, that is with $\mathcal{A}_{0} \neq \mathbb{C}$, and not for the bare (squeezed) white noise alone.

\section{§3.6. Formal derivation of the interaction operator}

In contrast to the foregoing investigations, we study here the interacting system in a more heuristic manner.

The invariance of our (squeezed) white noise state $\omega_{n, c}$ with respect to the shift $\sigma_{t}$ implies the existence of a strongly continuous unitary group $\exp \left\{-i t H_{f}\right\}, \quad t \in \mathbb{R}$, on $L^{2}(\mathcal{C}, \omega)$, which implements $\sigma_{t}$ and satisfies $\exp \left\{-i t H_{f}\right\} A \Omega=\sigma_{t}(A) \Omega$ for all $A \in \mathcal{C}$. Thus we have $H_{f} \Omega=0$ for the selfadjoint operator $H_{f}$, which is considered as the free evolution Hamiltonian for the photon system in the (squeezed) white noise. Hence for the composed system $\mathcal{A}=\mathcal{A}_{0} \otimes \mathcal{C}$ the free evolution is given by $S_{t}()=.\exp \left\{-i t\left(\mathbb{1} \otimes H_{f}\right)\right\}$. $\exp \left\{i t\left(\mathbb{1} \otimes H_{f}\right)\right\}$.

Now we are interested in the interacting dynamics $T_{t}$ on $\mathcal{A}$ associated with the additive cocycle $\beta_{t}=b_{t}+K t$ from Subsection 3.4, where $b_{t}$ is from (3.15) and $K$ is given by (3.17), resp. the unitary cocycle $u_{t}$ constructed from $\beta_{t}$ according to Equation (3.6).

But instead of treating the free and the interacting dynamics, $S_{t} \operatorname{resp} . T_{t}($. $=u_{t}^{*} S_{t}(.) u_{t}$, as $\mathrm{W}^{*}$-dynamical systems on the von Neumann algebra $\mathcal{A}$, here for simplicity we consider unitary time evolutions on the GNS-Hilbert space $L^{2}(\mathcal{A}, \varphi)$ similarily to traditional quantum mechanics. By $\Gamma$ it is denoted the interaction operator leading from the free evolution $\exp \left\{i t\left(\mathbb{1} \otimes H_{f}\right)\right\}$ to the interacting evolution $\exp \left\{i t H_{i}\right\}$ with the total Hamiltonian $H_{i}=\mathbb{1} \otimes H_{f}+$ $\Gamma$. Especially, we have $T_{t}()=.\exp \left\{-i t H_{i}\right\}$. $\exp \left\{i t H_{i}\right\}$ on $\mathcal{A}$ (cf., e.g., [12, Corollary 5.4.2]). From Equation (3.4) it follows that the interacting and the free unitary time evolutions are connected by the cocycle $u_{t}$ according to

$$
\exp \left\{i t H_{i}\right\}=\exp \left\{i t\left(\mathbb{1} \otimes H_{f}\right)\right\} u_{t} .
$$

This implies $u_{t}$ to describe the dynamics in the interaction picture: If $\Gamma(t):=$ $S_{t}(\Gamma)=\exp \left\{-i t\left(\mathbb{1} \otimes H_{f}\right)\right\} \Gamma \exp \left\{i t\left(\mathbb{1} \otimes H_{f}\right)\right\}$ is the free evolution of the interaction operator, then one gets the Schrödinger equation in the interaction picture as

$$
-i \frac{d}{d t} u_{t}=\Gamma(t) u_{t}
$$


Hence we conclude that the interaction operator is given by $\Gamma=-\left.i\left(d u_{t} / d t\right)\right|_{t=0}$. This formula is used for formally deducing the interaction operator $\Gamma$.

Observation 3.5. The interaction operator $\Gamma$ is formally given by

$$
\Gamma=H-\left[M^{*} \otimes a_{n, c}\left(i \delta_{0}\right)+M \otimes a_{n, c}^{*}\left(i \delta_{0}\right)\right],
$$

where $\delta_{0}$ is the delta function at the origin in $\mathbb{R}$, and $a_{n, c}\left(\delta_{0}\right)$ means a formal smearing of the annihilation operator of $\omega_{n, c}$ with the delta function $\delta_{0}$.

Formal Argumentation. From Theorem 3.1 we have $d u_{t}=d \beta_{t} \cdot u_{t}$ with $u_{0}=\mathbb{1}$. It holds $d \beta_{t}=d b_{t}+K d t$ with $d b_{t}$ from Equation (3.20) and $K=-(1 / 2) P_{0}\left(b_{1}^{*} b_{1}\right)+i H$. Thus with the explicit expression for $P_{0}\left(b_{1}^{*} b_{1}\right)$ according to Equation (3.18) we obtain in a formal sense

$$
\begin{aligned}
d u_{t}= & {\left[M^{*} \otimes d a_{n, c}(t)-M \otimes d a_{n, c}^{*}(t)+i H d t\right.} \\
& \left.+\frac{1}{2}\left(\bar{c} M M+c M^{*} M^{*}-n M M^{*}-(n+1) M^{*} M\right) d t\right] \cdot u_{t} \\
= & \exp \left\{M^{*} \otimes d a_{n, c}(t)-M \otimes d a_{n, c}^{*}(t)+i H d t\right\} \cdot u_{t} .
\end{aligned}
$$

Here we have used the multiplication rules of the Itô table from the previous Subsection, which especially imply that in the series expansion of the exponential only the terms up to the second order survive. Note that the exponential expression $d u_{t}=\exp \{\cdots\} \cdot u_{t}$ is of the same type for every (squeezed) white noise state $\omega_{n, c}$ contained in $\tilde{B}_{1}^{o}$, i.e., satisfying $n(n+1)>|c|^{2}$ according to Theorem 2.5. We formally conclude that $\left(d u_{t}\right) /\left.(d t)\right|_{t=0}=M^{*} \otimes\left(d a_{n, c}(t)\right) /\left.(d t)\right|_{t=0}-$ $M \otimes\left(d a_{n, c}^{*}(t)\right) /\left.(d t)\right|_{t=0}+i H$. But $\left(d a_{n, c}(t)\right) /\left.(d t)\right|_{t=0}=-a_{n, c}\left(\delta_{0}\right)$. Now observe $\Gamma=-\left.i\left(\left(d u_{t}\right) /(d t)\right)\right|_{t=0}$.

For more details to the interaction operator and how it arises from a physical point of view, we refer to [8], [9].

\section{Acknowledgement}

This work is part of a research project supported by the Deutsche Forschungsgemeinschaft.

\section{References}

[1] Accardi, L., Frigerio, A. and Lewis, J. T., Quantum stochastic processes, Publ. RIMS, Kyoto Univ., 18 (1996), 97-133. 
[2] Accardi, L. and Lu, Y. G., Wiener noise versus Wigner noise in quantum electrodynamics, Quantum Prob. Rel. Topics, VIII (1993), 1-18.

[3] — The Wigner semi-circle law in quantum electrodynamics, Comm. Math. Phys., 180 (1996), 605-632.

[4] Araki, H., On quasifree states of the canonical commutation relations II, Publ. RIMS, Kyoto Univ., 7 (1971/72), 121-152.

[5] Araki, H. and Shiraishi, M., On quasifree states of the canonical commutation relations I, Publ. RIMS, Kyoto Univ., 7 (1971/72), 105-120.

[6] Araki, H. and Woods, E. J., Representations of the canonical commutation relations describing a nonrelativistic infinite free Bose gas, J. Math. Phys., 4 (1963), 637-662.

[7] Arimitsu, T., A canonical formalism of dissipative quantum systems. Non-equilibrium thermo field dynamics, Condensed Matter Physics, 4 (1994), 26-88. (Ukrainian National Academy of Sciences).

[8] Barchielli, A., Measurement theory and stochastic differential equations in quantum mechanics, Phys. Rev. A, 34 (1986), 1642-1649.

[9] - Direct and heterodyne detection and other applications of quantum stochastic calculus to quantum optics, Quantum Opt., 2 (1990), 423-441.

[10] - Applications of quantum stochastic calculus to quantum optics, Quantum Prob. Rel. Topics, VI (1991), 111-125.

[11] Barnett, C., Streater, R. F. and Wilde, I. F., Quasifree quantum stochastic integrals for the CAR and CCR, J. Funct. Anal., 52 (1983), 19-47.

[12] Bratteli, O. and Robinson, D. W., Operator Algebras and Quantum Statistical Mechanics I, II, Springer, Berlin, Heidelberg, New York, 1987, 1981.

[13] Davies, E. B., Quantum Theory of Open Systems, Academic Press, London, New York, San Francisco, 1976.

[14] Ekert, A. K. and Knight, P. L., Relationship between semiclassical and quantummechanical input-output theories of optical response, Phys. Rev., A 43 (1991), 39343938.

[15] Evans, D. E. and Lewis, J. T., Dilations of Irreversible Evolutions in Algebraic Quantum Theory, Dublin, Communications of the Dublin Institute for Advanced Studies, Series A (Theoretical Physics), No. 24, 1977.

[16] Gardiner, C. W., Handbook of Stochastic Methods, Springer, Berlin, Heidelberg, New York, 1985.

[17] Gardiner, C. W. and Collett, M. J., Input and output in damped quantum systems: Quantum stochastic differential equations and the Master equation, Phys. Rev. A, 31 (1985), 3761-3774.

[18] Goswami, D. and Sinha, K. B., Hilbert modules and stochastic dilation of a quantum dynamical semigroup on a von Neumann algebra, Comm. Math. Phys., 205 (1999), 377-403.

[19] Hellmich, J., Köstler, C. and Kümmerer, B., Stationary Quantum Markov Processes as Solutions of Stochastic Differential Equations, Quantum Probability, Banach Center Publications, Vol. 43, Institute of Mathematics, Polish Academy of Sciences, Warszawa, 1998, pp. 217-229.

[20] Hertle, J. and Honegger, R., Limiting Gibbs states and dynamics for thermal photons, J. Math. Phys., 33 (1992), 143-151.

[21] - Rigorous derivation of Planck's law in the thermodynamic limit, J. Math. Phys., 33 (1992), 343-348.

[22] Hewitt, E. and Ross, K. A., Abstract Harmonic Analysis I, II, Springer, New York, Berlin, Heidelberg, Tokyo, 1963, 1970.

[23] Honegger, R., Decomposition of positive sesquilinear forms and the central decomposition of gauge-invariant quasi-free states on the Weyl algebra, Z. Naturforsch., 45a (1990), 17-28.

[24] — Globale Quantentheorie der Strahlung, Doctoral Thesis, Tübingen, 1991.

[25] Honegger, R. and Rieckers, A., Squeezing Bogoliubov transformations on the infinite 
mode CCR-algebra, J. Math. Phys., 37 (1996), 4292-4309.

[26] Honegger, R. and Rieckers, A., Squeezing of optical states on the CCR-algebra, Publ. RIMS, Kyoto Univ., 33 (1997), 869-892.

[27] - Squeezed variances of smeared Boson fields, Helv. Phys. Acta, 70 (1997), 507541.

[28] - Squeezing operations in Fock space and beyond, Physica A, 242 (1997), 423438.

[29] Huang, J. and Kumar, P., Photon-counting statistics of multimode squeezed light, Phys. Rev. A, 40 (1989), 1670-1673.

[30] Hudson, R. L. and Lindsay, J. M., Uses of non-Fock Quantum Brownian Motion and a Quantum Martingale Representation Theorem, in Quantum Probability and Applications II, pp. 276-305, Heidelberg 1984, Lecture Notes in Math., 1136, Springer-Verlag, 1985.

[31] Hudson, R. L. and Parthasarathy, K. R., Quantum Ito's formula and stochastic evolutions, Commun. Math. Phys., 93 (1984), 301-323.

[32] Kadison, R. V. and Ringrose, J. R., Fundamentals of the Theory of Operator Algebras II, Academic Press, New York, London, 1986.

[33] Kossakowski, A., Frigerio, A., Gorini, V. and Verri, M., Quantum detailed balance and KMS condition, Comm. Math. Phys., 57 (1977), 97-110.

[34] Kümmerer, B., Markov dilations on W*-algebras, J. Funct. Anal., 63 (1985), 193-177.

[35] —, Survey on a Theory of Non-Commutative Stationary Markov Processes, in Quantum Probability and Applications III, pp. 154-182, Oberwolfach 1987, Lecture Notes in Math., 1303, Springer-Verlag, 1988.

[36] - Stochastic Processes with Values in $M_{n}$ as Couplings to Free Evolutions, Tübingen, Preprint, 1993.

[37] Kümmerer, B. and Maassen, H., Elements of quantum probability, Quantum Prob. Comm., X (1998), 73-100.

[38] Lance, E. C., Hilbert $C^{*}$-Modules, London Math. Soc. Lecture Notes Ser., 210, Cambridge University Press, Cambridge, 1995.

[39] Loudon, R., The Quantum Theory of Light, Clarendon Press, Oxford, 1979.

[40] Loudon, R. and Knight, P. L., Squeezed light, J. Mod. Opt., 34 (1987), 709-759.

[41] Ma, X. and Rhodes, W., Multimode squeeze operators and squeezed states, Phys. Rev. A, 41 (1990), 4625-4631.

[42] Manuceau, J. and Verbeure, A., Quasi-free states of the C.C.R.-algebra and Bogoliubov transformations, Comm. Math. Phys., 9 (1968), 293-302.

[43] Nelson, E., Notes on non-commutative integration, J. Funct. Anal., 15 (1974), 103-116.

[44] Pedersen, G. K., C*-Algebras and their Automorphism Groups, Academic Press, London, New York, 1979.

[45] Petz, D., An Invitation to the Algebra of Canonical Commutation Relations, Leuven Notes in Mathematical and Theoretical Physics, 2, University Press, Leuven, 1990.

[46] Rieckers, A., Classical States of Quantized Boson Fields, Proceedings of the V International Conference on "Selected Topics in Quantum Field Theory and Mathematical Physics", Liblice, Czechoslovakia, June 1989, J. Niederle and J. Fischer, eds., World Scientific, Singapor, New Jersey, London, Hong Kong, 1990.

[47] Robinson, D. W., The ground state of the Bose gas, Comm. Math. Phys., 1 (1965), 159-174.

[48] Saito, T. and Arimitsu, T., A system of quantum stochastic differential equations in terms of non-equilibrium thermo field dynamics, J. Phys. A, 30 (1997), 7573-7595.

[49] Shale, D. and Stinespring, W. F., States on the Clifford algebra, Ann. Math., 80 (1964), $365-381$.

[50] Skeide, M., Hilbert modules in quantum electrodynamics and quantum probability, Comm. Math. Phys., 192 (1998), 569-604.

[51] Takesaki, M., Theory of Operator Algebras I, Springer, New York, Berlin, Heidelberg, 1979. 
[52] Terp, M., L ${ }^{p}$-Spaces Associated with von Neumann Algebras, Notes Københavns Universitet, Matematisk Institut, Rapport No. 3a/3b (1981).

[53] Testard, D., Les Etat Quasi-Libre du Champ des Bosons consideres comme Etats d'Equilibre à l'Aide de la Condition de Kubo, Martin et Schwinger, Thèse de Doctorat d'Etat. Marseille: Centre de Physique Théorique, Faculté des Sciences de Marseille, No. d'Ordre: A.O. 3680, 1970.

[54] Walls, D. F., Squeezed states of light, Nature, 306 (1983), 141-146.

[55] Yuen, H. P., Generalized coherent states and the statistics of two-photon-Lasers, Phys. Lett. A, 51 (1975), 1-2.

[56] - Two-photon coherent states of the radiation field, Phys. Rev. A, 13 (1976), 2226-2243.

[57] Yuen, H. P. and Shapiro, J. H., Optical communication with two-photon coherent states, part I, IEEE Trans. Inform. Theory, IT-24 (1978), 657-668.

[58] - Optical communication with two-photon coherent states, part II, Optics Letters, 4 (1979), 334.

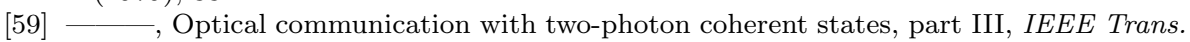
Inform. Theory, IT-26 (1980), 78-93. 